\title{
Tiny-scale molecular structures in the Magellanic Clouds
}

\section{FUSE, HST and VLT observations}

\author{
M. K. André ${ }^{1}$, F. Le Petit ${ }^{2,4}$, P. Sonnentrucker ${ }^{3}$, R. Ferlet ${ }^{1}$, E. Roueff ${ }^{2}$, T. Civeit ${ }^{1}$, J-.M. Désert ${ }^{1}$, \\ S. Lacour ${ }^{1}$, and A. Vidal-Madjar ${ }^{1}$ \\ 1 Institut d'Astrophysique de Paris, CNRS/UPMC, 98, Bd ARAGO, 75014 Paris, France \\ e-mail: andre@iap.fr \\ 2 LUTH and FRE2462 du CNRS, Observatoire de Paris, Place J. Jansen, 921995 Meudon Cedex, France \\ 3 Johns Hopkins University, Baltimore, Maryland, USA \\ ${ }^{4}$ Onsala Space Observatory, Onsala 439 92, Sweden
}

Received 18 December 2003 / Accepted 1 April 2004

\begin{abstract}
The objective of this series of two papers is to investigate small-scale molecular structures in the Magellanic Clouds (hereafter MCs). We report on the FUSE detections of the HD and CO molecules on the lines of sight towards three Large Magellanic stars: Sk-67D05, Sk-68D135, and Sk-69D246. HD is also detected for the first time on the lines of sight towards two Small Magellanic Cloud stars: AV 95 and Sk 159. While the HD and CO abundances are expected to be lower in the Large Magellanic Cloud where molecular fractions are a third of the Galactic value and where the photodissociation flux is up to thousands of times as large, we report an average $\mathrm{HD} / \mathrm{H}_{2}$ ratio of $1.4 \pm 0.5 \mathrm{ppm}$ and a $\mathrm{CO} / \mathrm{H}_{2}$ ratio ranging from 0.8 to $2.7 \mathrm{ppm}$ similar to the Galactic values. We tentatively identify a deuterium reservoir (hereafter D-reservoir) towards the Small Magellanic Cloud, along the light path to AV 95. We derive a D/H ratio ranging from $1 . \times 10^{-6}$ to $1.1 \times 10^{-5}$. Combining FUSE and HST/STIS data we also analyzed the $\mathrm{H}_{2}, \mathrm{Cl}$ I, Cl II, Fe II, S II, C I, C I*, and C I** content, when available. High resolution VLT observations of Na I, Ca II, and Ca I were obtained in support of the lower resolution FUSE and STIS data for three targets in order to unravel the sightline velocity structures. These observations form the only such set of detections in the Magellanic Clouds to date and allow us to investigate in detail some of the physical properties of the intervening molecular gas. Our detection of the HD and CO molecules in the Magellanic Clouds is an argument for the existence of dense $\left(n_{\mathrm{H}}>100 \mathrm{~cm}^{-3}\right)$ components. Furthermore, we demonstrate that these components are probably extremely small molecular clumps (possibly as small as $10^{-2} \mathrm{pc}$ ) or filaments similar to the tiny-scale atomic structures (TSAS) recently observed in the halo of our Galaxy by Richter et al. (2003). For these five sightlines, we also detected molecular hydrogen originating in the Galactic disk. From these observations we conclude that tiny-scale molecular filamentary structures are present in the disk of the Galaxy as well.
\end{abstract}

Key words. ISM: molecules - galaxies: abundances

\section{Introduction}

Through the years, the study of molecular clouds through the $\mathrm{CO}$ emission lines has greatly improved our knowledge of the physical properties within the Magellanic Clouds themselves and helped constrain the theoretical models of molecular cloud formation and chemistry in different Galactic environments (Cohen et al. 1988). Over the past decade, in particular, the Swedish-ESO-Submillimeter-Telescope (SEST) has been used to map the radio-emission of molecular complexes within the LMC at an 8"'0 angular resolution, which is an order of magnitude higher than in previous surveys. Such resolution corresponds to a linear extent of about $10 \mathrm{pc}$ at the LMC distance of $50 \mathrm{kpc}$. The latter survey led to the clear detection of small CO clumps with sizes possibly smaller than $10 \mathrm{pc}$ (Lequeux et al. 1994; Garay et al. 2002), typical densities of a few thousand molecules per cubic centimeter and virial masses around $10^{4}$ solar masses. However, smaller clumps and AUscale structures, such as those tentatively identified in absorption in the Milky Way (Frail et al. 1994; Lauroesch et al. 2000; Richter et al. 2003) are still out of reach.

An alternative method relies on detailed spectroscopic analyses of selected sightlines that might actually help to unravel such small-scale structures in the Magellanic InterStellar Medium (MC ISM). The high-resolution spectroscopic abundance studies of Welty et al. (1999) based on the observations of SN 1987A by Vidal-Madjar et al. (1987) showed that the MC ISM exhibits properties similar to those of the warm, low-density Galactic disk clouds and can contain complex velocity structures with more than 20 components. Some of the absorbers observed toward SN 1987A also showed signatures of relatively cool gas $(T<1500 \mathrm{~K})$. The lack of information concerning the molecular content precluded further characterization of this cool phase, however. 
With the FUSE data it is now possible to investigate the molecular content of this cool phase also via $\mathrm{H}_{2}, \mathrm{CO}$, and HD. Although the HD molecule is the most abundant molecule in the interstellar medium after $\mathrm{H}_{2}$, relatively few observations have been reported to date in the Galaxy and few comparisons with theoretical models of translucent clouds have been done (Le Petit et al. 2002). Similarly to $\mathrm{H}_{2}$, the presence of HD molecule is best determined through its ground state transitions which only occur in the FUV domain. The detection is however difficult due to a low molecular abundance $\left(\mathrm{HD} / \mathrm{H}_{2} \approx\right.$ $\left.10^{-6}\right)$. The ground-state transitions of HD were first observed with Copernicus in the local ISM towards bright, nearby stars (Spitzer \& Cochran 1973; Wright \& Morton 1979). Only recently did the FUSE mission (Moos et al. 2000) re-open the FUV window with sufficiently high sensitivity to allow the detection of HD further away in the Galactic disk (Ferlet et al. 2000; Rachford et al. 2002; Lacour et al. 2004 in prep.). As to extragalactic search for HD, only two detections were found in the literature, one in the LMC by Bluhm \& de Boer (2001) with the FUSE satellite, the other in a Damped Lyman Alpha system towards PKS1232+0.82 (Varshalovich et al. 2001). The conclusions from the work of Bluhm \& de Boer (2001) are consistent with the existence of a cool phase in the LMC with molecular abundances $\left(\mathrm{H}_{2}, \mathrm{HD}, \mathrm{CO}\right)$ typical of Galactic translucent sightlines (Rachford et al. 2002).

The aim of this series of two papers is to provide a more extensive knowledge of the "dense", small-scale molecular structures within the Magellanic Clouds. We wish to point out that in this work we specifically use the term "dense" to designate gas with proton density, $n_{\mathrm{H}}$, above $100 \mathrm{~cm}^{-3}$. In the present paper, emphasis is put on the analysis of $\mathrm{H}_{2}, \mathrm{HD}, \mathrm{CO}, \mathrm{Cl}$ I, Cl II, Na I, Ca II, S II, Fe II, C I, C I*, and C I**, when available, towards five bright Magellanic Cloud stars. Sk-67D05, Sk-68D135 and Sk-69D246 are located in the LMC. AV 95, and Sk 159 are in the SMC. In Sect. 2, we describe the reduction of the FUSE, VLT and STIS datasets. The analysis methods used to derive the species column densities are described in Sect. 3. Individual conclusions for each target are presented in Sect. 4. A preliminary discussion of these results is given in Sect. 5 and Sect. 6 summarizes our study.

\section{Observations}

\subsection{FUSE observations}

With the help of the web-based archive maintained at the Institut d'Astrophysique de Paris $^{1}$ we have searched for HD lines among the 55 LMC sightlines and 26 SMC sightlines observed with FUSE (see the spectral atlas by Danforth et al. 2002). Each dataset was reprocessed with the CALFUSE pipeline version 2.0.5. The reduction and calibration procedure includes thermal drift correction, geometric distortion correction, heliocentric velocity correction, dead time correction, wavelength and flux calibrations (Sahnow et al. 2000).

The full observation of a given star is often divided into several sub-exposures which are usually aligned by crosscorrelation over the entire wavelength domain. However, due

\footnotetext{
${ }^{1}$ More at http://fuse.iap.fr
}

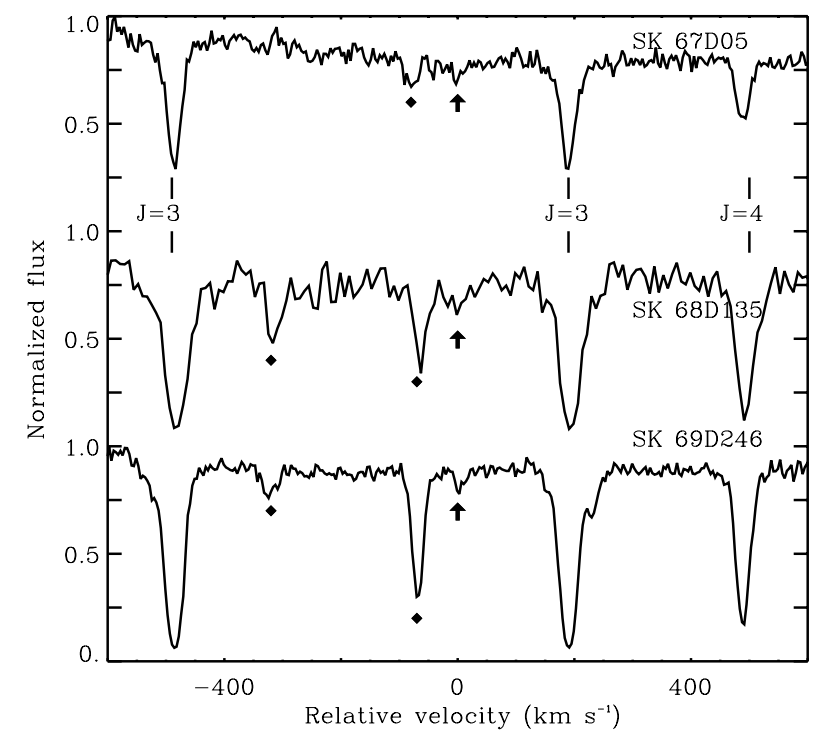

Fig. 1. Detection of the $1043 \AA \mathrm{HD}(J=0)$ line in the LMC. $\mathrm{H}_{2}$ Rotational levels 3 and 4 are indicated for the LMC component while the black diamonds point to the strongest $\mathrm{H}_{2}$ features from the Galactic component. The velocities are shifted so that the $\lambda 1043 \AA$ HD line appears at $0 \mathrm{~km} \mathrm{~s}^{-1}$.

to the time-dependent stretching of the wavelength calibration between exposures, coaddition of the entire spectrum at once may lead to systematic shifts in the final coadded spectrum. In other words, even though the reference absorption features are perfectly aligned at one end of the spectrum, the global coaddition could result in random shifts at the other end. To avoid these effects and optimize the coaddition process with the FUSE data we divided each subexposure into $10 \AA$ windows and performed the coaddition within these spectral domains, weighting each domain by its exposure time. No coaddition of data from different channels was performed. We however used each channel, independently, in the profile fitting procedure. As a byproduct we computed a basic two-components velocity structure $(\mathrm{MW}+\mathrm{MC})$ and overplotted a model of the molecular species on top of each spectrum in order to detect the weak $\mathrm{HD}$ lines at the MCs velocities.

Finally, out of the $81 \mathrm{MC}$ sightlines observed only five showed detectable amounts of HD; three detections occurred in the LMC (Fig. 1) and two detections occurred in the SMC (Fig. 2). Note that HD is possibly present in a few others lines of sight but could not be detected because of insufficient $S / \mathrm{N}$ ratios or large amounts of Galactic molecular hydrogen crowding the HD lines arising from the MCs. In Table 1, we report the main stellar and interstellar parameters for each of the five stars. A summary of the FUSE observations is given in Table 2.

\subsection{VLT observations}

Spectra of the three LMC targets were obtained at the Very Large Telescope Kueyen at Paranal, Chile, in 2003 April using the Ultraviolet Echelle Spectrograph (UVES, Dekker et al. 2000) in visitor mode (see Table 3). The red and blue arm of UVES, respectively centered at $3900 \AA$ and $8600 \AA$, 
Table 1. Lines-of-sight properties and references.

\begin{tabular}{lrrlcc}
\hline \hline & RA & Dec & Spectral & & \\
LMC/SMC stars & $\mathrm{J} 2000$ & $\mathrm{~J} 2000$ & Type & $E_{(B-V)}{ }^{a}$ & References \\
\hline Sk-67D05 & 45018.96 & -673937.90 & O9.7Ib & 0.16 & 1,2 \\
Sk-68D135 & 53748.60 & -68558.00 & ON9.7 Ia+ & 0.27 & 1,3 \\
Sk-69D246 & 53853.50 & -6920.70 & WN6h & 0.10 & 1,4 \\
AV 95 & 05121.54 & -724412.90 & O7 III & 0.06 & 1 \\
Sk 159 & 11558.84 & -732124.10 & B0.5 Iaw & 0.09 & 1 \\
\hline
\end{tabular}

${ }^{a}$ Total color excess (MW+LMC).

${ }^{1}$ Tumlinson et al. (2002).

${ }^{2}$ Friedman et al. (2000).

${ }^{3}$ Ehrenfreund et al. (2002).

${ }^{4}$ Bluhm \& De Boer (2001).

Table 2. Log of FUSE observations ${ }^{a}$.

\begin{tabular}{lcccc}
\hline \hline LMC/SMC stars & Dataset & Date & $\begin{array}{c}\text { Exposure time }^{b} \\
\text { Ksecs }\end{array}$ & $\begin{array}{c}S / N \\
\text { per pixel }\end{array}$ \\
\hline Sk-67D05 & $P 1030703$ & $2000-10-07$ & 3.5 & 5 \\
& $P 1030704$ & $2000-10-04$ & 4.0 & 6 \\
Sk -68D135 & $P 1173901$ & $2000-02-12$ & 6.8 & 5 \\
Sk -69D246 & $P 1031802$ & $1999-12-16$ & 22.1 & 9 \\
AV 95 & $P 1150404$ & $2000-05-31$ & 13.8 & 7 \\
Sk 159 & $P 1030501$ & $2000-10-11$ & 6.9 & 5 \\
\hline
\end{tabular}

${ }^{a}$ All these targets have been observed in Time-Tagged (TTAG)mode through the large aperture (LWRS).

${ }^{b}$ After sorting for bad data.

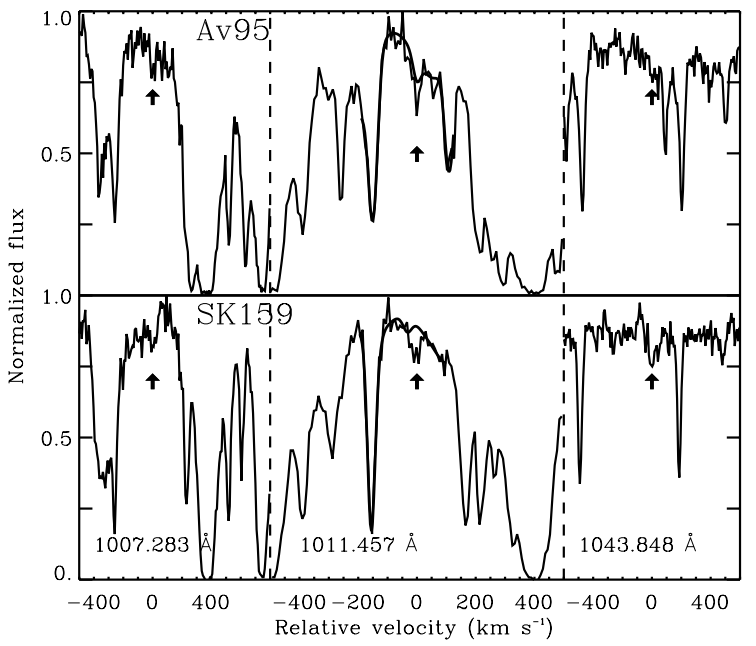

Fig. 2. Detections of the HD molecule towards the SMC at three different wavelengths. AOD analysis is based on the $1007 \AA$ HD transition. For clarity we overplotted the profile fitting model over the $1011 \AA$ transition which is blended with a Galactic $\mathrm{H}_{2}$ line. The velocities are shifted so that the HD lines appear at $0 \mathrm{~km} \mathrm{~s}^{-1}$ in each case.

were used at the same time in dichroic mode during the same night. A non-standard setup was used with the red arm centered at $7500 \AA$. We used the $0{ }^{\prime} 4$ slit to achieve a resolving power of 110000 (2.6 pixels). With this setup we observed the
Na I (5890 ̊̊) doublet, Ca I (4230 ̊), and the Ca II (3930 ̊) doublet. 1-D spectra were extracted with the UVES pipeline (Ballester et al. 2000). A careful examination of the corrected 2D images showed that the background and the potential stray light were well corrected with the standard pipeline procedure. However, we noted a shift of the order of $14 \mathrm{~km} \mathrm{~s}^{-1}$ in the wavelength calibration which was corrected using the telluric $\mathrm{NaI}$ emission lines (located at $0 \mathrm{~km} \mathrm{~s}^{-1}$ geocentric velocity). $S / N$ ratios of about 30 per pixel were achieved in about $20 \mathrm{~min}$.

To remove the telluric lines that contaminate the spectra around the Na I doublet (5890 ̊), we took an exposure of the well known early type, unreddened star $\alpha$ Vir. We normalized the continuum of all the spectra to unity. We finally divided the normalized LMC spectra by the normalized $\alpha$ Vir spectrum. The absence of telluric lines in the final spectra shown in Figs. 3-5 is indicative of the effectiveness and accuracy of our method.

\subsection{STIS observations}

One LMC star (Sk-69D246) and one SMC star (AV 95) were previously observed with the HST/STIS instrument using the $140 \mathrm{M}$ grating and MAMA detectors (Woodgate et al. 1998; Kimble et al. 1998). The observations contain a complete wavelength coverage from $1150 \AA$ to $1730 \AA$ at 45800 resolving power (2.0 pixels). The $S / N$ ratios 
Table 3. Log of VLT/UVES observations obtained on the 27th May 2003*.

\begin{tabular}{lrlccc}
\hline \hline Begin (UT) & Object & Exposure time (s) & Air Mass Start & Air Mass End & Setup \\
\hline 23:26 & Sk-69D246 & 1200 & 1.69 & 1.76 & Standard Dichroic \\
23:50 & Sk-68D135 & 1200 & 1.78 & 1.86 & Standard Dichroic \\
$00: 16$ & Sk-67D05 & 1200 & 2.14 & 2.28 & Standard Dichroic \\
$00: 42$ & Sk-68D135 & 489 & 2.02 & 2.06 & Non Standard Red \\
$01: 07$ & Sk-69D246 & 900 & 2.16 & 2.27 & Non Standard Red \\
01:29 & Sk-67D05 & 513 & 2.79 & 2.89 & Non Standard Red \\
\hline
\end{tabular}

* Observations collected at the European Southern Observatory, Paranal, Chile [ESO VLT 69.A-0123(A)].

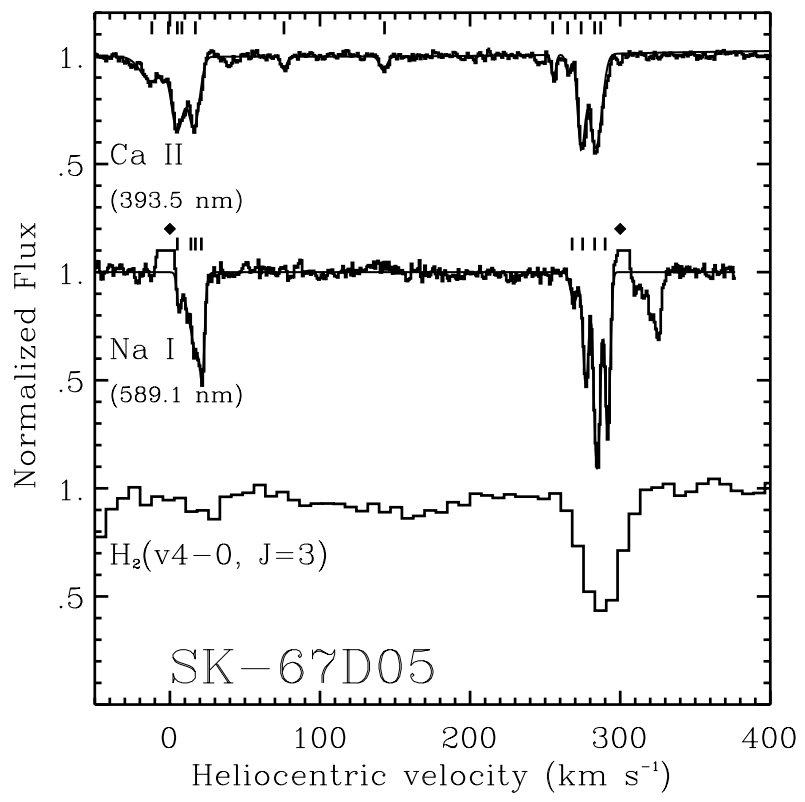

Fig. 3. VLT/UVES data for $\mathrm{Na}$ and $\mathrm{Ca}$ shows up to 14 components, 5 of them arising in the MW. Two distincts groups of absorbers show up in the spectra: galactic at $\approx+10 \mathrm{~km} \mathrm{~s}^{-1}$ and LMC at $\approx+290 \mathrm{~km} \mathrm{~s}^{-1}$. Only two weak intermediate velocity absorbers are detected in Ca II. $\mathrm{Na}$ I airglows have been truncated and indicated with diamonds. Note that the fits of $\mathrm{NaI}$ have been performed on both lines of the doublet. Part of the second doublet is seen in the far left of the displayed spectrum.

per pixel are about 15 and 10 for Sk-69D246 and AV 95, respectively. We used these medium-resolution $\left(6.5 \mathrm{~km} \mathrm{~s}^{-1}\right)$ echelle observations to derive the column densities of C I, C I*, $\mathrm{CI}^{* *}, \mathrm{Cl} \mathrm{I}$, and $\mathrm{S} \mathrm{II}$ in both sightlines. A summary of the STIS observations is given in Table 4 .

\section{Line-of-sight velocity structure}

With the exception of the pathlength towards Sk 159 for which little information is available, the detailed interstellar component velocity structure is inferred from high resolution $\mathrm{NaI}$, $\mathrm{Ca}$ II, or $\mathrm{Cl}$ I spectra and is given in the heliocentric reference frame. The use of different types of tracers allowed us to better differentiate between cold molecular or purely diffuse atomic components.

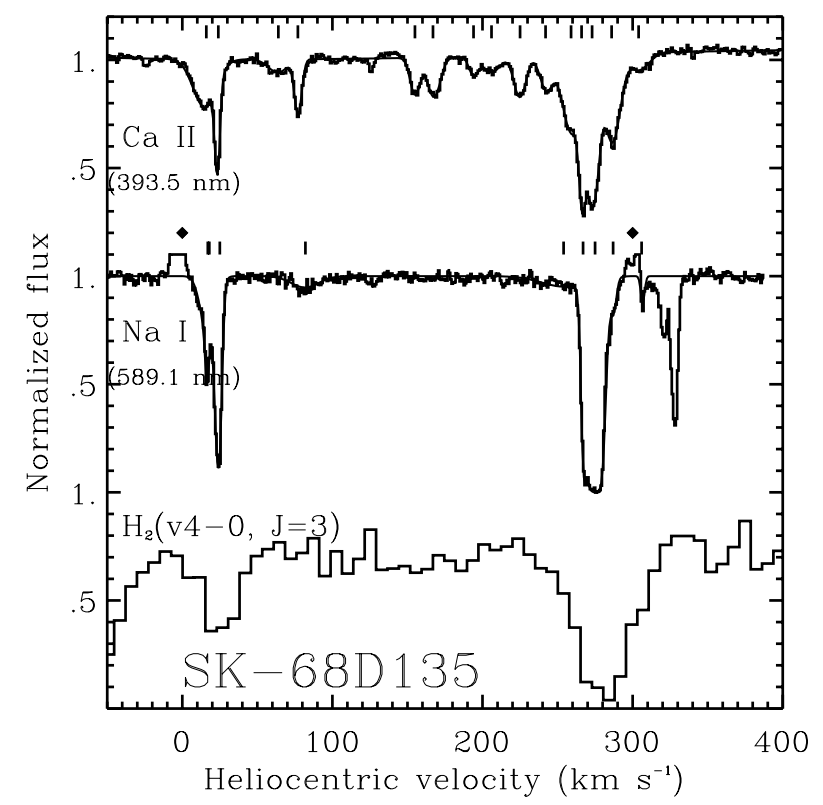

Fig. 4. The sightline towards Sk-68D135 is similar to the sightline towards Sk-69D246 with two groups of strong Na I absorbers: galaxy and LMC. The FUSE molecular data are consistent with a single molecular absorber in the LMC but the resolution is not sufficient to discard the existence of a more complex structure. Note the HVC Na I and $\mathrm{Ca}$ II components around $+310 \mathrm{~km} \mathrm{~s}^{-1}$. $\mathrm{Na}$ I airglows have been truncated and indicated with diamonds.

\subsection{Diffuse gas tracers: Ca II, Ca I, and Na I}

We observed the three LMC targets with the VLT with a resolving power of 110000 around $589 \mathrm{~nm}, 423 \mathrm{~nm}$, and $393 \mathrm{~nm}$. These observations gave us access to three important species that are usually used in combination to derive the physical conditions of a sightline in the ISM.

The NaI/Ca II ratio for instance can be used as a tracer of the integrated depletion (Welty et al. 1999). Typical values for this ratio in the warm diffuse medium are of the order of -2.0 dex where the $\mathrm{Ca}$ is not depleted. In colder, denser clouds (ignoring the shocks), two processes compete to drastically change the $\mathrm{Ca}$ II abundance: a severe depletion of calcium onto the grains (by about $2.0 \mathrm{dex}$ ) and the decrease of the ionization radiation field that makes of $\mathrm{Ca}$ II the dominant ionization stage (Pottasch 1972; Bertin et al. 1993; Welty et al. 1996). In general, in such cold medium the $\mathrm{NaI} / \mathrm{Ca}$ II is found 
Table 4. Log of HST/STIS observations.

\begin{tabular}{lcccc}
\hline \hline Star & ID & Date & Exposure (s) & Aperture \\
\hline Sk-69D246 & 06LZ12010 & $2002-11-13$ & 1200 & $0.2 \times 0.2$ \\
AV 95 & 04WR17010 & $1999-05-16$ & 2448 & $0.2 \times 0.2$ \\
& 04WR17020 & $1999-05-16$ & 3168 & $0.2 \times 0.2$ \\
\hline
\end{tabular}

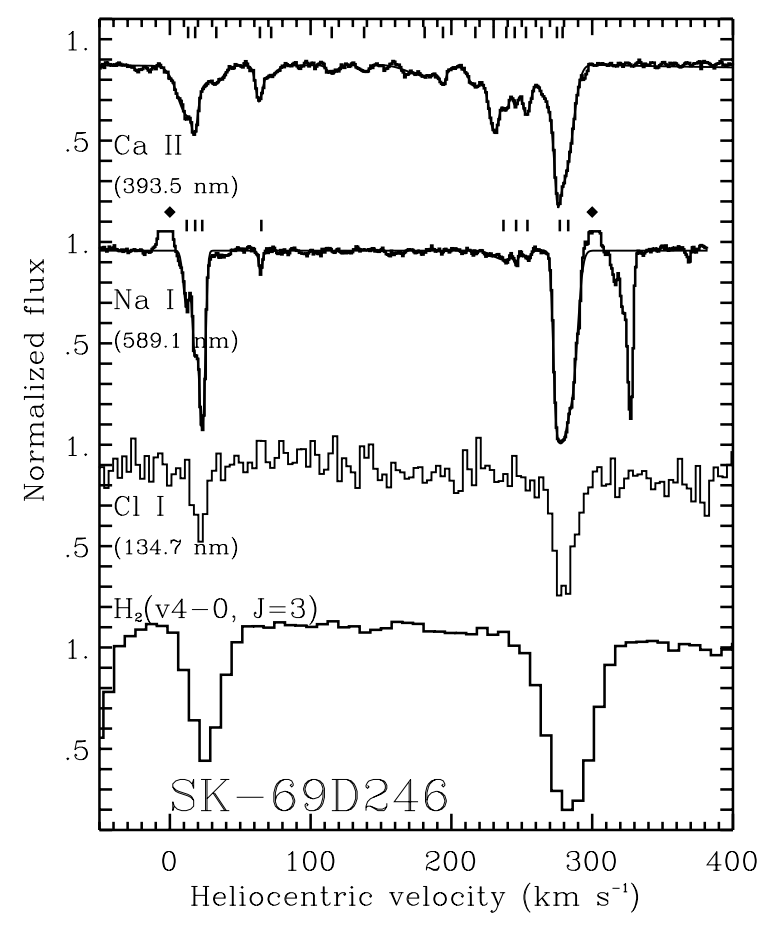

Fig. 5. With 19 components this sightline has the most complicated velocity structure of the sample. With VLT/UVES, STIS and FUSE data, this sightline is also the one for which we have the most complete information about the velocity structure. The last $2 \mathrm{CaII}, \mathrm{NaI}$ and $\mathrm{H}_{2}$ components coincide more or less in the profile fitting. Na I airglows have been truncated and indicated with diamonds.

to be as high as $2.5 \mathrm{dex}$ and more. We use this ratio to discriminate between possible diffuse atomic or denser molecular components in the VLT data, thus better constraining the location of the molecular absorbers seen with FUSE at lower resolution.

CaI is clearly detected towards one LMC target: $\mathrm{Sk}-69 \mathrm{D} 246$. In the cooler neutral gas phase where $\mathrm{Ca}$ II is the dominant ion stage, the $\mathrm{Ca} / \mathrm{Na}$ I ratio can be a useful indicator of the temperature. For $T<3000 \mathrm{~K}, \log N(\mathrm{Ca} \mathrm{I}) / N(\mathrm{NaI})$ is of the order of -0.1 . At temperatures lower than $1500 \mathrm{~K}$, $\log N(\mathrm{Ca} \mathrm{I}) / N(\mathrm{Na} \mathrm{I})$ is in between -2.5 and -4.5 (Welty et al. 2001). Moreover, if we assume that the ionization equilibrium is reached in the plasma we can, in principle, use the ratio $N(\mathrm{Ca} \mathrm{I}) / N(\mathrm{Ca}$ II $)$ to get a direct estimate of $n_{\mathrm{e}}$. For the calcium atoms, the relation is simply given by (Welty et al. 1999): $N(\mathrm{Ca} \mathrm{I}) / N(\mathrm{Ca}$ II $)=n_{\mathrm{e}} /(\Gamma / \alpha)$ where $\Gamma$ is the photoionization rate and $\alpha$ is the recombination coefficient.

\subsection{Molecular gas tracers: $\mathrm{Cl}$}

Chlorine possesses a unique chemistry in which $\mathrm{Cl}$ II reacts rapidly with $\mathrm{H}_{2}$ when the latter is optically thick to form $\mathrm{HCl}^{+}$, which in turn leads to Cl I and H I (Jura 1974; Jura \& York 1978). Therefore, chlorine is predominantly ionized in H I regions while it is predominantly neutral when cold, optically thick $\mathrm{H}_{2}$ components are present (Sonnentrucker et al. 2002, 2003).

So far, only four $\mathrm{Cl}$ I lines have experimentally determined $f$-values (Schectman et al. 1993). Two of these lines (1347 and $1363 \AA$ ) appear in the STIS range and the other two (1088 and $1097 \AA$ ) in the FUSE range. We have positive detections of $\mathrm{Cl}$ I at $1088 \AA$ for all three LMC stars. Because the $1097 \AA$ line in the LMC gas is often blended with the Galactic components our $\mathrm{Cl}$ I column density estimate relies on the $1088 \AA$ line alone for Sk-67D05, Sk-68D135 and Sk 159. The $1347 \AA$ line was additionally used to constrain the $\mathrm{Cl}$ I column densities towards the two other stars for which archival STIS data exist.

Cl II exhibits only two transitions, both in the FUSE range at $1063 \AA$ and $1071 \AA$. The $1063 \AA$ line is often completely obliterated by molecular hydrogen, and hence unusable. The $1071 \AA$, on the other hand, shows only mild-to-moderate blending with an adjacent $\mathrm{H}_{2}(J=4)$ line and can therefore be used depending on the sightline complexity. However, the continuum around the latter line is lowered by a broad wind feature. This results in a reduced $S / N$ ratio in that particular spectral region of the spectrum for both the LMC and SMC stars. We therefore only report one secure detection of this line towards Sk -69D246 and an upper limit towards Sk -67D05.

\section{Analysis}

\subsection{Profile fitting (PF)}

Because of the velocity overlap between the Galactic and the MC features and the low resolution of the FUSE data, many absorption lines are blended. Extensive use of profile fitting methods is therefore mandatory to account for these blends.

The profile fitting program, called Owens, is a Fortran procedure developed by the FUSE french PI team and is one of the most suitable for studying FUV data (Lemoine et al. 2002). The great advantage of this routine is its ability to fit different spectral domains and various species simultaneously. Thus a good $b$-value estimate can be gained from the comparison of the Doppler broadenings of heavy and light species while at the same time blends are resolved naturally. In practice, however, the atomic and molecular species are rarely distributed in a similar way over the different components and great caution 
Table 5. Summary of lines used in this work.

\begin{tabular}{|c|c|c|c|}
\hline Species & $\begin{array}{c}\text { Wavelength } \\
(\AA)\end{array}$ & $f$-value & Dataset \\
\hline CI & 1276.4822 & $0.449 \times 10^{-2}$ & STIS \\
\hline C I & 1277.2452 & $0.932 \times 10^{-1}$ & STIS \\
\hline C I* & 1276.7498 & $0.252 \times 10^{-2}$ & STIS \\
\hline C I* & 1277.2827 & $0.705 \times 10^{-1}$ & STIS \\
\hline C I* & 1277.5131 & $0.223 \times 10^{-1}$ & STIS \\
\hline C I* & 1279.0562 & $0.736 \times 10^{-3}$ & STIS \\
\hline C I* & 1279.8907 & $0.126 \times 10^{-1}$ & STIS \\
\hline CI** & 1277.1899 & $0.273 \times 10^{-3}$ & STIS \\
\hline $\mathrm{CI} \mathrm{I}^{* *}$ & 1277.5500 & $0.791 \times 10^{-1}$ & STIS \\
\hline C I** & 1277.7233 & $0.155 \times 10^{-1}$ & STIS \\
\hline $\mathrm{CI} \mathrm{I}^{* *}$ & 1277.9539 & $0.817 \times 10^{-3}$ & STIS \\
\hline C I** & 1279.2290 & $0.378 \times 10^{-2}$ & STIS \\
\hline $\mathrm{CI} \mathrm{I}^{* *}$ & 1279.4980 & $0.199 \times 10^{-3}$ & STIS \\
\hline $\mathrm{CaI}$ & 4227.9180 & $0.175 \times 10^{-1}$ & VLT \\
\hline $\mathrm{Ca} I \mathrm{II}$ & 3934.7750 & $0.629 \times 10^{0}$ & VLT \\
\hline $\mathrm{Ca} I \mathrm{II}$ & 3969.5901 & $0.312 \times 10^{0}$ & VLT \\
\hline $\mathrm{ClI}$ & 1088.0590 & $0.810 \times 10^{-1}$ & FUSE \\
\hline $\mathrm{ClI}$ & 1347.2396 & $0.153 \times 10^{0}$ & STIS \\
\hline $\mathrm{Cl}$ II & 1071.035 & $0.150 \times 10^{-1}$ & FUSE \\
\hline $\mathrm{Fe} I I$ & 1125.4478 & $0.160 \times 10^{-1}$ & FUSE \\
\hline $\mathrm{Fe}$ II & 1127.0984 & $0.282 \times 10^{-2}$ & FUSE \\
\hline $\mathrm{Fe}$ II & 1143.2260 & $0.177 \times 10^{-1}$ & FUSE \\
\hline Fe III & 1122.5240 & $0.544 \times 10^{-1}$ & FUSE \\
\hline $\mathrm{NaI}$ & 5891.5833 & $0.641 \times 10^{0}$ & VLT \\
\hline $\mathrm{NaI}$ & 5897.5581 & $0.320 \times 10^{0}$ & VLT \\
\hline HD Lyman $(\mathrm{V} 14-0, J=0)$ & 959.8174 & $0.147 \times 10^{-1}$ & FUSE \\
\hline HD Werner $(\mathrm{V} 0-0, J=0)$ & 1007.2830 & $0.325 \times 10^{-1}$ & FUSE \\
\hline HD Lyman $(\mathrm{V} 8-0, J=0)$ & 1011.4570 & $0.262 \times 10^{-1}$ & FUSE \\
\hline HD Lyman $(\mathrm{V} 5-0, J=0)$ & 1042.8480 & $0.206 \times 10^{-1}$ & FUSE \\
\hline HD Lyman $(\mathrm{V} 3-0, J=0)$ & 1066.2710 & $0.115 \times 10^{-1}$ & FUSE \\
\hline $\mathrm{CO}(\mathrm{C}-\mathrm{X}, J=0)$ & 1087.8669 & $0.619 \times 10^{-1}$ & FUSE \\
\hline $\mathrm{CO}(\mathrm{C}-\mathrm{X}, J=1)$ & 1087.9590 & $0.206 \times 10^{-1}$ & FUSE \\
\hline $\mathrm{CO}(\mathrm{C}-\mathrm{X}, J=1)$ & 1087.8209 & $0.413 \times 10^{-1}$ & FUSE \\
\hline $\mathrm{CO}(\mathrm{C}-\mathrm{X}, J=2)$ & 1088.0040 & $0.247 \times 10^{-1}$ & FUSE \\
\hline $\mathrm{CO}(\mathrm{C}-\mathrm{X}, J=2)$ & 1087.7739 & $0.371 \times 10^{-1}$ & FUSE \\
\hline $\mathrm{CO}(\mathrm{C}-\mathrm{X}, J=3)$ & 1088.0480 & $0.265 \times 10^{-1}$ & FUSE \\
\hline $\mathrm{CO}(\mathrm{C}-\mathrm{X}, J=3)$ & 1087.7260 & $0.354 \times 10^{-1}$ & FUSE \\
\hline $\mathrm{CO}(\mathrm{C}-\mathrm{X}, J=4)$ & 1088.0919 & $0.275 \times 10^{-1}$ & FUSE \\
\hline $\mathrm{CO}(\mathrm{C}-\mathrm{X}, J=4)$ & 1087.6780 & $0.344 \times 10^{-1}$ & FUSE \\
\hline
\end{tabular}

must be used when grouping species together within a single component.

Profile fitting of the atomic and molecular species was thus performed in two independent steps since the atomic absorbers usually show much more complicated velocity structures and larger $b$-values (more details are given in subsequent sections). Also, data from the different instruments were used at the same time in order to fit simultaneously different species and constrain the different contributions to the Doppler broadening (temperature and turbulent velocity). Table 5 is a summary of selected atomic and molecular transitions used in this work.

The other advantage of the Owens routine is that it allows one to treat as parameters the instrument background, the Point Spread Function (PSF) and possible shifts between spectral windows due to residual errors in the wavelength calibration.

In particular, special care must be taken in the choice of the PSF when working with the FUSE data. Despite many attempts to characterize the FUSE PSF (Hébrard et al. 2002; 


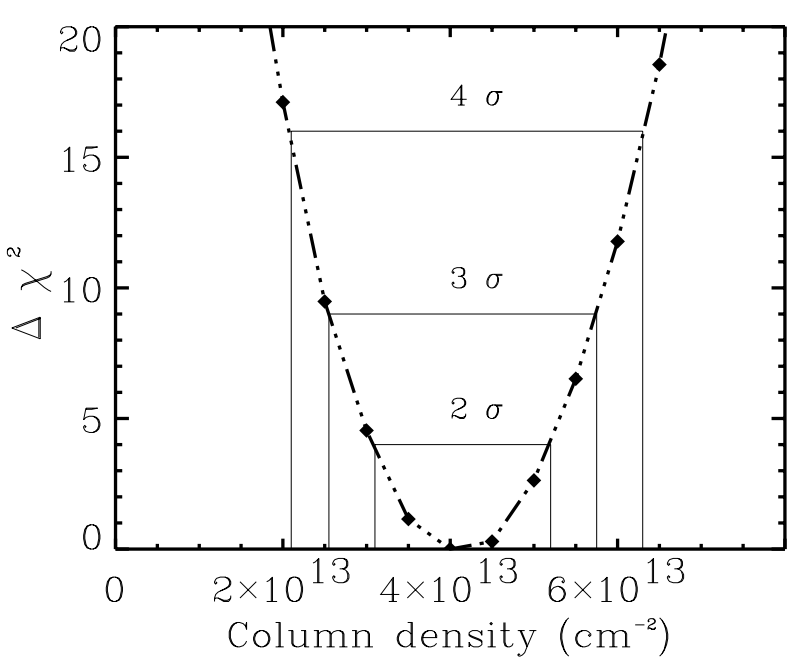

Fig. 6. $\chi^{2}$ plot from the profile fitting of HD towards Sk-69D246. $2 \sigma$ error bars are calculated with the standard $\Delta \chi^{2}$ method. For this fit the $\overline{\chi^{2}}$ is very close to unity.

Wood et al. 2002), its true shape is elusive and seems to vary with time. Current values found in the FUSE literature range from a quite optimistic LWRS FWHM of $15 \mathrm{~km} \mathrm{~s}^{-1}$ to $25 \mathrm{~km} \mathrm{~s}^{-1}$. In our case, we have to coadd invidual exposures of the same channel and the composite PSF relies on the quality of the coaddition process as well. However, because the $S / N$ of the final data is relatively low, we found that a simple Gaussian PSF with $20 \mathrm{~km} \mathrm{~s}^{-1}$ (resolution of 15000) $F W H M$ worked well, and various tests with FWHM $10 \%$ larger or $10 \%$ smaller showed that the PSF plays a minor role in the final error budget largely dominated by the errors in the $b$-value. Throughout this paper we thus assume the PSF to be independent of the dataset and the observation date.

The errors were derived with the standard $\chi^{2}$ method as described in Hébrard et al. (2002) and as shown in Fig. 6. We want to stress that, in most of the fits performed, the converged $\overline{\chi^{2}}$ is close to unity indicating both that the fit is consistent with the data and that the data error vector is well estimated by the FUSE pipeline version 2.0.5. However, when the errors are dominated by systematics, a more conservative method needs to be em-

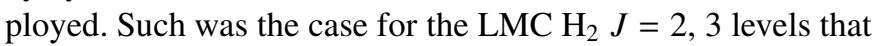
are dominated by small errors in the $b$-value. For these, we explored the different $b$-values consistent with the global fit and report the full range of possible values.

\subsection{Apparent Optical Depth (AOD)}

Both AOD and profile fitting techniques were used to determine $N(\mathrm{HD})$ and $N(\mathrm{Cl} \mathrm{I})$ in order to better understand potential sources of systematic error. The AOD method (Savage \& Sembach 1991) yields apparent column densities, $N_{\mathrm{a}}$, that are equivalent to the true column density if no unresolved saturated structure is present. If such structure is present, then $N_{\mathrm{a}}<N_{\mathrm{PF}}$, and the apparent column density is a lower limit to the true column density. Our AOD analysis, including estimation of the errors, follows the procedures outlined in Savage $\&$ Sembach (1991). Note that it was not possible to apply the
AOD technique to $\mathrm{CO}$ because of a severe blend with one $\mathrm{H}_{2} J=4$ Galactic line. This blend was resolved naturally using the profile fitting technique.

\section{3. $\mathrm{H}_{2}$ column densities}

We adopted the wavelengths, oscillator strengths, and damping constants for the molecular hydrogen transitions from Abgrall et al. (1993a) for the Lyman system and Abgrall et al. (1993b) for the Werner system. The inverse values of the total radiative lifetimes are reported in Abgrall et al. (2000). The profile fitting was performed both in the MW and the MCs over 10 to 20 different spectral regions simultaneously. Rotational levels up to $J=6$ were considered.

In the MCs, the $J=0$ and $J=1$ levels are the most populated levels and show damping wings. The estimates of the total $\mathrm{H}_{2}$ column densities in the MCs are therefore well constrained by these two levels. The higher $J$ levels, on the other hand, appear to show various degrees of saturation. Thus, prior knowledge of the velocity structure in the line of sight is mandatory for the analysis of these levels. This preliminary analysis was performed using higher resolution $\left(3 \mathrm{~km} \mathrm{~s}^{-1}\right)$ VLT optical data of Na I, Ca II that trace the most diffuse components, and STIS high resolution data of $\mathrm{Cl}$ I that trace the optically thick $\mathrm{H}_{2}$ components. We find that with the exception of Sk-69D246 and Sk 159, the present sightlines are dominated by a single molecular absorber.

The errors were derived following the procedure described in Sect. 3.1. Note however that while cold unresolved components might well still be present in the FUSE spectra, this does not affect our conclusions based on $J=0$ and $J=1$ lines. The total $\mathrm{H}_{2}$ column density and the kinetic temperature of this gas $\left(T_{01}\right)$ still remain accurately determined.

All of the molecular absorbers detected in the Galaxy have column densities several orders of magnitude smaller than towards the Magellanic Clouds. This is obviously an observational bias due to the severe blending between the Galactic and the Magellanic features. An upper limit to the Galactic $\mathrm{H}_{2}$ is of the order of $\log N\left(\mathrm{H}_{2}\right) \approx 18.0$ dex. Beyond that limit, Magellanic absorption lines start to suffer from the strong MW blending.

\subsection{HD column density}

All the ground state ro-vibrational bands of the HD molecules lie below $1180 \AA$. The FUSE range is therefore perfectly suited to study this molecule. The wavelengths for the $J=0$ and 1 rotational levels were taken from Roueff \& Zeippen (1999) while the $f$-values come from the calculations of Abgrall \& Roueff (2003). This new set of improved $f$-values takes into account the rotational coupling between electronic states.

In the LMC, because of the severe blending of most of the HD lines (shifted by about $+250 \mathrm{~km} \mathrm{~s}^{-1}$ ) with strong $\mathrm{H}_{2}$ features from the Milky Way, we could only select a handful of useful spectral domains containing $\operatorname{HD}(J=0)$ : around $959 \AA$, $1011 \AA, 1043 \AA$ and $1066 \AA$. Yet, depending on the $S / N$ ratio of a given spectrum and depending on the particular blending 
between Galactic species and HD along a given sightline, some of these domains had to be discarded for the analysis. As an example, profile fitting of $\mathrm{HD}$ in the spectrum of Sk-69D246 is shown in Fig. 5.

Our method of investigation combines AOD measurements of the strongest detected transition (HD $J=0$ at $1043 \AA$ ) with profile fitting performed simultaneously over several spectral domains. Both results are indeed comparable and demonstrate that the HD lines are on the linear part of the curve of growth. Thus, the HD column is not sensitive to the $b$-value used in the profile fitting method. Each of these methods allows us to get independent measurements, with their own error estimates, that are then compared and averaged.

The situation is the same with HD lines originating in the SMC with the difference that the typical velocity shift is only $+150 \mathrm{~km} \mathrm{~s}^{-1}$. Towards the SMC there are only three available HD lines clear of blending, at $1007 \AA$, $1011 \AA$, and $1043 \AA$. Again, the final result is the average of profile fitting and AOD measurements.

For the sightline towards Sk-69D246, we compared our result with the work of Bluhm \& de Boer (2001) towards the LMC. Our HD column density appears to be only half of their claimed value. While they used only 2 transitions of the HD molecule (the $1043 \AA$ and $1066 \AA$ lines) assuming no blending, we fitted 2 additional transitions at $1011 \AA$ and $959 \AA$ with FUSE. Performing simultaneous fits over several windows, we were able to unravel a blend of the $1066 \AA$ line. Indeed, the equivalent width measured at $1066 \AA$ is twice as big as expected from the measurement of the equivalent width at $1043 \AA$. To our knowledge, this extra absorption feature does not correspond to any known species either at the MW or at the LMC velocity. In the end we attributed this blend to a stellar line (see Fig. 7). This blend was therefore responsible for an overestimation of the HD column density published by Bluhm $\&$ de Boer (2001).

\section{5. $\mathrm{CO}$}

Two strong ${ }^{12} \mathrm{CO}$ bands are usually detected in the FUSE spectra, the $E-X$ band at $1076 \AA$ and the ${ }^{12} \mathrm{CO} C-X$ band centered at $1087 \AA$. We detected the $1087 \AA$ band in the spectra of the LMC sightlines while we tentatively derived upper limits for the SMC sightlines. The other bands present in the spectra could not be used either because they are too weak or because of blends with a stronger Galactic feature. The $f$-values were taken from Morton \& Noreau (1994) for the $1087 \AA$ A line.

The ${ }^{13} \mathrm{CO}$ absorption lines are also located in the FUSE range and are totally blended with the ${ }^{12} \mathrm{CO}$ absorption lines. However, we did not add the ${ }^{13} \mathrm{CO}$ bands in our profile fitting since the ${ }^{13} \mathrm{C}$ isotopes are supposed to be a minute fraction of the total number of carbon atoms. It is well known that the ${ }^{12} \mathrm{C} /{ }^{13} \mathrm{C}$ ratio is a sensitive indicator of the degree of stellar nucleosynthesis and is expected to decrease with the Galactic chemical evolution (Woosley \& Weaver 1995). Since the ${ }^{12} \mathrm{CO} /{ }^{13} \mathrm{CO}$ ratio is of the order of 70 in the Galaxy (Savage et al. 2002) it is clear that ${ }^{13} \mathrm{CO}$ is negligible at the level of accuracy of our analysis.

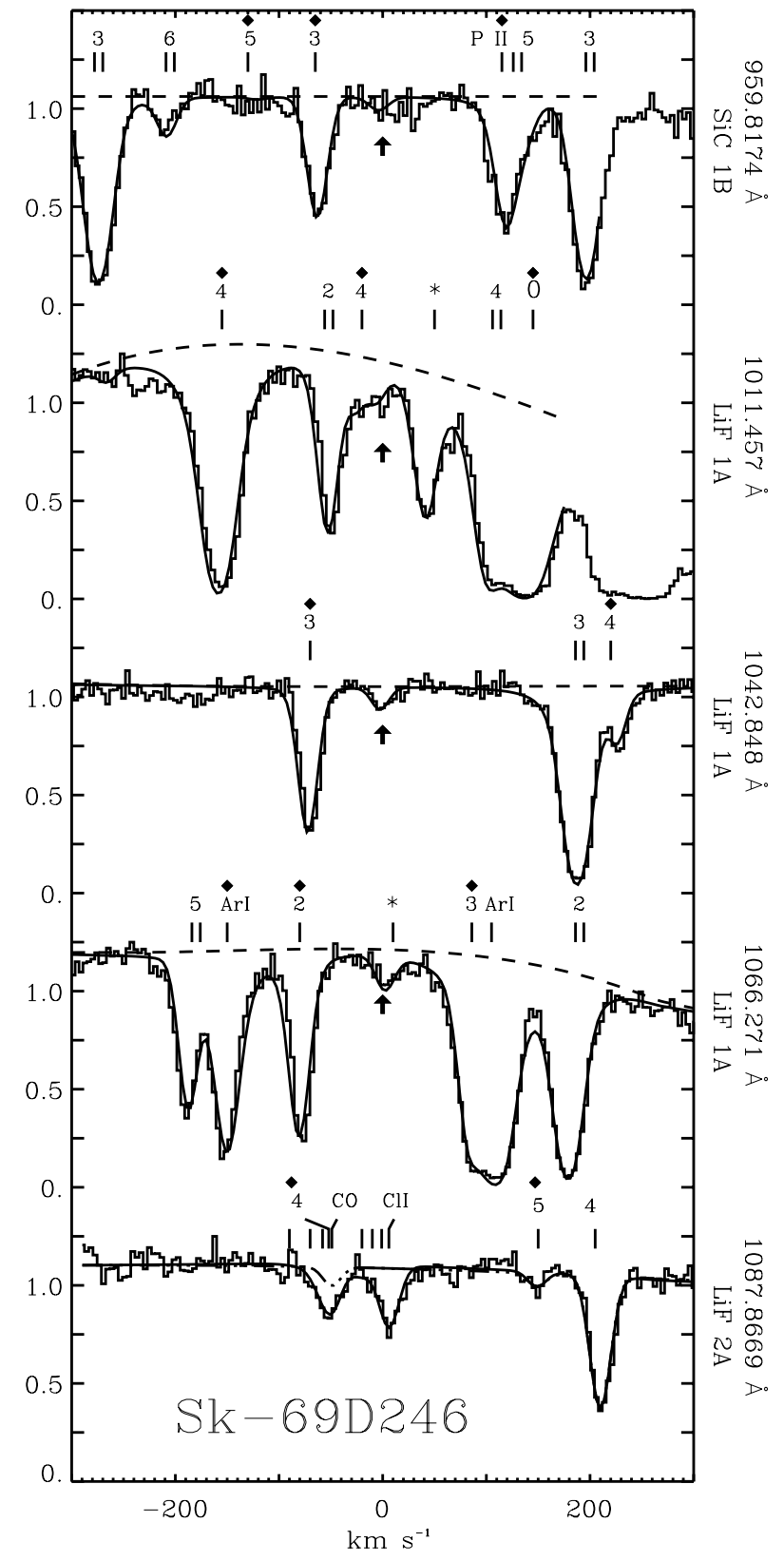

Fig. 7. Examples of profiles fitted on the FUSE data for Sk -69D246. Galactic absorption lines are indicated by black diamonds and the unidentified stellar features by asteriks. The spectra are centered on the LMC HD lines except for the last plot which is centered on the LMC ClI line. Note the $J=4$ Galactic absorption over the profile of the LMC CO absorption. The numerous CO rotational levels (not labeled) spreading over $100 \mathrm{~km} \mathrm{~s}^{-1}$.

Our fits include rotational levels up to $J=3$. Despite the weakness of the band we conducted an investigation of the individual population distribution. The $\mathrm{CO}$ rotational levels spread over $100 \mathrm{~km} \mathrm{~s}^{-1}$ in the $C-X$ band. It is hence possible to partially deblend their different contribution to the profile with the FUSE resolution $\left(\approx 20 \mathrm{~km} \mathrm{~s}^{-1}\right)$. Rotational levels greater than $J=4$ are found to be negligible, which is consistent with the subthermal excitation temperatures $(\approx 4 \mathrm{~K})$ measured in the diffuse Galactic disk (Rachford et al. 2002). 
Table 6. Logarithmic column densities of carbon for two sightlines.

\begin{tabular}{lcc}
\hline \hline & Sk-69D246 & AV 95 \\
\hline & Galaxy & Galaxy \\
\hline $\mathrm{C} \mathrm{I}$ & $13.40_{-0.10}^{+0.08}$ & $13.64_{-0.60}^{+0.60}$ \\
$\mathrm{C} \mathrm{I}^{*}$ & $13.16_{-0.18}^{+0.08}$ & $13.37_{-0.25}^{+0.25}$ \\
$\mathrm{C} \mathrm{I}^{* *}$ & $12.95_{-0.12}^{+0.15}$ & $12.78_{-0.18}^{+0.22}$ \\
$f_{1}{ }^{a}$ & $0.30 \pm 0.15$ & $0.32 \pm 0.26$ \\
$f_{2}{ }^{a}$ & $0.18 \pm 0.10$ & $0.08 \pm 0.24$ \\
$n_{\mathrm{H}}$ range & {$[60 \ldots 600]$} & {$[30 \ldots 600]$} \\
\hline & LMC & SMC \\
\hline $\mathrm{C} \mathrm{I}^{b}$ & $13.85_{-0.06}^{+0.06}$ & $12.90_{-0.20}^{+0.27}$ \\
$\mathrm{CI}^{*}$ & $13.72_{-0.10}^{+0.10}$ & $12.95_{-0.18}^{+0.25}$ \\
$\mathrm{CI}^{* *}$ & $13.44_{-0.06}^{+0.05}$ & $12.70_{-0.40}^{+0.20}$ \\
$f_{1}{ }^{a}$ & $0.35 \pm 0.07$ & $0.41 \pm 0.07$ \\
$f_{2}{ }^{a}$ & $0.18 \pm 0.10$ & $0.23 \pm 0.28$ \\
$n_{\mathrm{H}}$ range $^{c}$ & {$[300 \ldots . .600]$} & {$[100 \ldots 800]$} \\
\hline
\end{tabular}

${ }^{a} f_{1}=N\left(\mathrm{CI}^{*}\right) / N\left(\mathrm{CI}_{\text {total }}\right)$ and $f_{2}=N\left(\mathrm{C} \mathrm{I}^{* *}\right) / N\left(\mathrm{C} \mathrm{I}_{\text {total }}\right)$.

$b$ The total density is derived from the model of Jenkins \& Shaya (1979) assuming the average Galactic radiation field.

${ }^{c}$ Same as ${ }^{b}$ but assuming 10 times the average Galactic radiation field.

Note that an additional difficulty towards the LMC stars resides in the partial blending of the $1087 \AA$ line with a Galactic $\mathrm{H}_{2} J=4$ line and a $\mathrm{Cl}$ I line. Fortunately the analysis of the Galactic $\mathrm{H}_{2}$ and $\mathrm{Cl}$ I makes it possible to safely deblend the LMC CO absorption lines when present.

\section{6. $C l, C I^{*}$, and $C I^{\star \star}$}

CI absorption lines are present in all our FUSE spectra. However, given that most of these lines are multiplets and that the $S / N$ ratio is not high enough to deblend the absorption lines, it is not possible to derive the relative population of the excited states. The situation is worsened by the potential blend between Galactic and MC C I absorption lines.

In the case of Sk-69D246 and AV 95, however, the blending can be resolved due to the existence of complementary STIS data that also contain a wealth of carbon lines at higher resolution (see Table 5). We estimate the column densities for the carbon lines and use them to derive the average density of the probed clouds.

The relative population of neutral carbon atoms in excited fine-structure levels of the ground electronic state can be used to estimate the number densities and temperatures of neutral clouds in the ISM (Jenkins \& Shaya 1979; Jenkins \& Tripp $2001)$. Using the $f_{1}-f_{2}$ diagram $\left(f_{1}=N\left(\mathrm{CI}^{*}\right) / N\left(\mathrm{CI}_{\text {total }}\right)\right.$; $\left.f_{2}=N\left(\mathrm{CI}^{* *}\right) / N\left(\mathrm{CI}_{\text {total }}\right)\right)$, we thus derive the thermal pressures $(P / k)$ towards AV 95 and Sk-69D246. Assuming that the carbon lines arise in a dense molecular component with a temperature $T=T_{01}\left(\mathrm{H}_{2}\right)$ we can infer the average $\mathrm{H}$ number density, $n_{\mathrm{H}}$ of the medium crossed (see Table 6 and sections below).

\section{Results for individual sightlines}

\section{1. $S k-67 D 05$}

Sk-67D05 is a 09.7Ib star (Neubig et al. 1999; Tumlinson et al. 2002) located near the Western edge of the LMC. It lies in a diffuse H II region (Chu et al. 1994) with relatively low diffuse X-ray emission (Snowden \& Petre 1994). The sightline towards Sk-67D05 was the first LMC target investigated with FUSE showing a large amount of molecular hydrogen (Friedman et al. 2000). Fits of the Na I and Ca II lines reveal up to 12 components in four distinct groups as shown in Fig. 3: the Galactic group centered at $0 \mathrm{~km} \mathrm{~s}^{-1}$, an Intermediate Velocity Cloud (IVC) at $+77 \mathrm{~km} \mathrm{~s}^{-1}$, and High Velocity Cloud (HVC) at $+144 \mathrm{~km} \mathrm{~s}^{-1}$, and the LMC group centered at $+280 \mathrm{~km} \mathrm{~s}^{-1}$. Our VLT data show the hidden complexity behind the simple picture derived at lower resolution (15000) by Friedman et al. (2000) using FUSE data alone. A detailed summary of these $\mathrm{NaI}$ and $\mathrm{Ca}$ II components is given in Table 7.

The lack of $\mathrm{NaI}$ absorbers between the IVC and HVC groups is quite remarkable for an LMC target since large absorptions at $\approx+60 \mathrm{~km} \mathrm{~s}^{-1}$ and $\approx+120 \mathrm{~km} \mathrm{~s}^{-1}$ are usually observed along LMC sightlines. However, in their survey of Magellanic sightlines with IUE, Savage \& de Boer (1981) reported the detection of an IVC and a HVC cloud in the light path to Sk-67D05 through low ion absorptions (Mg I, O I, Si II, and Fe II). Danforth et al. (2002) recently confirmed the galactic origin of these absorbers from a deep investigation of dozens of FUSE LMC sightlines. They demonstrated that both the IVC and the HVC are located in the general direction of the Eastern edge of the LMC so that the Western line of sight towards Sk -67D05 is likely sampling only a small fraction of this material.

Molecular hydrogen absorption lines are detected in the FUSE data. Rotational levels up to $J=3$ were detected in the MW group and up to $J=6$ in the LMC group. Because some of the molecular hydrogen absorption lines are expected to be saturated, it is mandatory to use as much information as possible on the velocity structure of the molecular components. Although we do not have direct information from molecular tracer species with our high resolution VLT data, the detailed investigation of the $\mathrm{NaI}$ and the Ca II lines is used to infer a plausible molecular velocity structure. Firstly, using the $\mathrm{Na}$ I lines alone, we can constrain the position of the molecular absorbers to the position of the neutral atoms (as seen in $\mathrm{NaI}$ ) and compare the model with the FUSE data. In any case, we shall expect to see at most the same number of molecular components as of atomic components. However, when the separation between components is less than $10 \mathrm{~km} \mathrm{~s}^{-1}$ (well below the FUSE resolution) the profile fitting of the FUSE spectra is less reliable. Secondly, we can use the Na I/Ca II ratio to determine which atomic component is cool and dense enough to harbor molecules. Typically, the greater the ratio the more likely the presence of dust grains and hence molecules (Welty et al. 1996).

Among the possible NaI MW absorbers, two were found to match the velocity of the FUSE $\mathrm{H}_{2}$ absorption feature: one component at $+18 \mathrm{~km} \mathrm{~s}^{-1}$ and another component 
Table 7. Ca II and Na I component parameters towards the LMC.

\begin{tabular}{|c|c|c|c|c|c|c|c|}
\hline Star & Comp & $\begin{array}{c}V_{\text {hel }} \\
\text { Ca II }\end{array}$ & $\begin{array}{c}N_{10} \\
10^{10} \mathrm{~cm}^{-2}\end{array}$ & $\begin{array}{c}b \\
\mathrm{~km} \mathrm{~s}^{-1}\end{array}$ & $\begin{array}{l}V_{\text {hel }} \\
\mathrm{NaI}\end{array}$ & $\begin{array}{c}N_{11} \\
10^{11} \mathrm{~cm}^{-2}\end{array}$ & $\begin{array}{c}b \\
\mathrm{~km} \mathrm{~s}^{-1}\end{array}$ \\
\hline \multirow[t]{14}{*}{ Sk-67D05 } & 1 & -11 & 35.5 & 11.2 & & & \\
\hline & 2 & 0 & 8.6 & 3.8 & & & \\
\hline & 3 & 6 & 31.5 & 2.3 & 6 & 0.7 & 0.4 \\
\hline & 4 & 9 & 17.8 & 1.7 & & & \\
\hline & 5 & & & & 15 & 3.2 & 5.8 \\
\hline & 6 & 18 & 56.8 & 4.8 & 18 & 1.2 & 2.2 \\
\hline & 7 & & & & 22 & 2.6 & 0.8 \\
\hline & 8 & 77 & 4.8 & 2.3 & & & \\
\hline & 9 & 144 & 6.2 & 3.0 & & & \\
\hline & 10 & 256 & 6.5 & 0.7 & & & \\
\hline & 11 & 266 & 5.3 & 1.4 & 269 & 0.9 & 2.9 \\
\hline & 12 & 275 & 54.5 & 3.0 & 274 & 3.5 & 1.4 \\
\hline & 13 & 284 & 62.8 & 3.5 & 284 & 18.0 & 1.6 \\
\hline & 14 & 288 & 13.9 & 3.1 & 291 & 8.5 & 1.2 \\
\hline \multirow[t]{17}{*}{$\mathrm{Sk}-68 \mathrm{D} 135$} & 1 & 15 & 71.6 & 10.4 & 19 & 1.7 & 0.5 \\
\hline & 2 & 23 & 46.2 & 1.6 & 20 & 3.9 & 6.4 \\
\hline & 3 & & & & 27 & 12.2 & 1.8 \\
\hline & 4 & 63 & 146.0 & 8.5 & & & \\
\hline & 5 & 76 & 26.2 & 2.9 & & & \\
\hline & 6 & & & & 84 & 1.2 & 12.7 \\
\hline & 7 & 154 & 23.0 & 4.3 & & & \\
\hline & 8 & 166 & 31.7 & 5.6 & & & \\
\hline & 9 & 193 & 9.9 & 3.9 & & & \\
\hline & 10 & 205 & 16.0 & 7.5 & & & \\
\hline & 11 & 224 & 31.8 & 5.4 & & & \\
\hline & 12 & 241 & 27.3 & 5.6 & & & \\
\hline & 13 & 258 & 102.0 & 8.3 & 256 & 1.9 & 21.1 \\
\hline & 14 & 265 & 65.1 & 2.2 & 269 & 139. & 0.8 \\
\hline & 15 & 272 & 160.0 & 4.9 & 277 & 75.1 & 4.5 \\
\hline & 16 & 285 & 93.9 & 6.6 & 289 & 0.8 & 1.9 \\
\hline & 17 & 303 & 20.6 & 9.2 & 308 & 0.7 & 0.2 \\
\hline \multirow[t]{18}{*}{$S k-69 D 246$} & 1 & 14 & 86.5 & 9.9 & 14 & 1.9 & 2.5 \\
\hline & 2 & 19 & 14.6 & 1.9 & 20 & 3.5 & 1.6 \\
\hline & 3 & & & & 25 & 34.1 & 1.3 \\
\hline & 4 & 34 & 17.6 & 6.6 & & & \\
\hline & 5 & 65 & 18.6 & 2.9 & 67 & 0.4 & 0.3 \\
\hline & 6 & 73 & 9.2 & 5.3 & & & \\
\hline & 7 & 116 & 6.1 & 4.4 & & & \\
\hline & 8 & 139 & 5.1 & 4.3 & & & \\
\hline & 9 & 182 & 30.4 & 17.4 & & & \\
\hline & 10 & 195 & 6.3 & 2.3 & & & \\
\hline & 11 & 218 & 24.6 & 6.8 & & & \\
\hline & 12 & 231 & 66.3 & 5.2 & & & \\
\hline & 13 & 240 & 22.3 & 2.9 & 239 & 0.9 & 10.4 \\
\hline & 14 & 246 & 18.9 & 2.6 & 248 & 0.2 & 0.2 \\
\hline & 15 & 254 & 40.4 & 4.5 & 256 & 0.2 & 2.0 \\
\hline & 16 & 265 & 19.8 & 5.0 & & & \\
\hline & 17 & 276 & 42.3 & 1.7 & 279 & 46.1 & 3.1 \\
\hline & 18 & 280 & 217.0 & 7.8 & 285 & 17.4 & 5.5 \\
\hline
\end{tabular}

at $+22 \mathrm{~km} \mathrm{~s}^{-1}$. We then performed a two components analysis of the MW molecular content and find a total $\mathrm{H}_{2}$ column density of $\log N\left(\mathrm{H}_{2}\right)=15.24 \pm 0.17 \mathrm{dex}$, summed over the $J=0-3$ states (Table 8 ), while the rotational temperature $T_{01}$ is about $303 \mathrm{~K}$. These properties are typical of diffuse molecular clouds found in the halo as observed by Richter et al. (1999). It should be noted that our column density estimates for the individual rotational levels in the MW molecular component are somewhat larger than the estimates of Friedman et al. (2000). 
Table 8. Logarithmic column densities of molecular hydrogen derived in the MW.

\begin{tabular}{lccccc}
\hline \hline & Sk-67D05 & Sk-68D135 & Sk-69D246 & AV 95 & Sk 159 \\
\hline$J=0$ & $14.40_{-0.30}^{+0.15}$ & $17.84_{-0.05}^{+0.11}$ & $18.40_{-0.04}^{+0.04}$ & $18.00_{-0.06}^{+0.06}$ & $15.70_{-0.22}^{+0.30}$ \\
$J=1$ & $15.11_{-0.11}^{+0.22}$ & $18.08_{-0.04}^{+0.05}$ & $18.46_{-0.04}^{+0.04}$ & $17.84_{-0.08}^{+0.08}$ & $16.17_{-0.10}^{+0.30}$ \\
$J=2$ & $14.30_{-0.40}^{+0.24}$ & $16.60_{-0.10}^{+0.35}$ & $16.30_{-0.07}^{+0.10}$ & $17.00_{-0.15}^{+0.18}$ & $14.78_{-0.08}^{+0.43}$ \\
$J=3$ & $<14.70$ & $15.08_{-0.08}^{+0.42}$ & $15.70_{-0.08}^{+0.08}$ & $16.00_{-0.08}^{+0.08}$ & $\ldots$ \\
$J=4$ & $\ldots$ & $13.90_{-0.43}^{+0.28}$ & $14.23_{-0.06}^{+0.07}$ & $13.90_{-0.43}^{+0.30}$ & $\ldots$ \\
Total & $15.30 \pm 0.17$ & $18.29 \pm 0.05$ & $18.73 \pm 0.04$ & $18.26 \pm 0.05$ & $16.31 \pm 0.25$ \\
$T_{01}(\mathrm{~K})$ & 136 & 103 & 83 & 66 & 153 \\
$b_{\text {Dopp }}\left(\mathrm{km} \mathrm{s}^{-1}\right)$ & $2.9 ; 2.4^{a}$ & 4.8 & 4.6 & 1.5 & 2.1 \\
\hline
\end{tabular}

${ }^{a}$ Two molecular components are detected towards Sk $-67 \mathrm{D} 05$ at $+35 \mathrm{~km} \mathrm{~s}^{-1}$ and $+39 \mathrm{~km} \mathrm{~s}^{-1}$. We quote the total $\mathrm{H}_{2}$ column densities.

There are four atomic components spanning $30 \mathrm{~km} \mathrm{~s}^{-1}$ seen at the location of the LMC velocities. Imposing the 4-component solution on the FUSE data showed that the main LMC molecular absorber is associated with the atomic component at $291 \mathrm{~km} \mathrm{~s}^{-1}$ while less than $2 \%$ of the $\mathrm{H}_{2}$ arises in the other components. We thus assume that the LMC molecular absorption lines detected in FUSE are from this single component. We find an $\mathrm{H}_{2}$ column density of $\log N\left(\mathrm{H}_{2}\right)=19.44 \pm 0.05$ dex. With the exception of the $J=3$ measurement, all of our estimates of the molecular hydrogen rotational levels in the LMC component are consistent with the survey by Tumlinson et al. (2002). We thus similar temperatures with $T_{01} \approx 57 \mathrm{~K}$ and $T_{23} \approx 225 \mathrm{~K}$. The population of the rotational levels might then be used to infer the incident radiation field upon this LMC absorber.

In the following, we assume that the incident flux is responsible for the population of the rotational levels $J>2$. This is consistent with the observation of two excitation temperatures for the $\mathrm{H}_{2}$ component. The two temperatures are interpreted as the signatures of different processes acting together to populate the $\mathrm{H}_{2}$ molecule from the lower levels all the way up. The levels $J=0-2$ are predominantly populated by collisional processes, with typical excitation temperatures below $100 \mathrm{~K}$. In the Galactic disk, the population of higher rotational levels is explained by the conjugated effect of collisions, UV pumping, and formation processes (Spitzer \& Zweibel 1974). However, in the LMC the radiation field is likely the main source of excitation of these higher rotational levels. Thus from the balance of the $J=4$ level we can derive a direct estimate for the incident flux using the method developed in Wright \& Morton (1979).

The basic equilibrium equation can be translated in the following manner:

$p_{4} \cdot\left(N_{0}+N_{2}+N_{4}\right)=N_{J=4} \cdot A_{4}+N_{4}$

where $N_{2}$ is the number of photo-excited $\mathrm{H}_{2}$ molecules in $J=$ $2, p_{4}$ is the probability that photo-excited molecules cascade into $J=4$ and $A_{4}$ is the rate of radiative decay from $J=4$ to $J=2$. The number of photo-excited $\mathrm{H}_{2}$ molecules in a given rotational level is proportional to the number of available photons multiplied by the probability of being absorbed:

$N_{4}=\int_{J=4}\left(\frac{U_{\lambda} c \mathrm{~d} \lambda}{h v}\right) \times\left(\frac{I_{\lambda 0}-I_{\lambda}}{I_{\lambda 0}}\right)$.

If we assume that $U_{\lambda} / h v$ is constant over the far ultraviolet range and equal to $\left(U_{\lambda} / h v\right)_{1000}$, then the previous equations transform into:

$N_{4}=\left(\frac{U_{\lambda} c}{h v}\right)_{1000} \times \Sigma_{J=4} W_{\lambda}$

and

$p_{4} \cdot\left(\frac{U_{\lambda} c}{h v}\right)_{1000} . \Sigma_{J=0,2,4} W_{\lambda}=N_{J=4} \cdot A_{4}+\left(\frac{U_{\lambda} c}{h v}\right)_{1000} . \Sigma_{J=4} W_{\lambda}$

with $p_{4}=0.26$ and $A_{4}=2.76 \times 10^{-9} \mathrm{~s}^{-1}$. The total equivalent widths $\left(\Sigma W_{\lambda}\right)$ are then computed from models of the different rotational levels based on our measurements. Solving these equations yields $U_{\lambda} \approx 1.9 \times 10^{-16} \mathrm{erg} \mathrm{cm}^{-3} \AA^{-1}$ which is about three times the assumed Galactic value of $\approx 7 \times$ $10^{-17} \mathrm{erg} \mathrm{cm}^{-3} \AA^{-1}$ (see Draine 1978; Parravano et al. 2003).

Next, with the incident flux we can estimate the distance of the cloud to the background star. The large aperture of the FUSE satellite camples a $30^{\prime \prime} \times 30^{\prime \prime}$ sky area which represents about $8 \mathrm{pc} \times 8 \mathrm{pc}$ at the distance of the LMC. Since we do not see any other hot stars in the FUSE aperture, we assume that the background star is the main source of photons onto the cloud. Thus, following Bluhm \& de Boer (2001), we calculate the distance using the simple cross product $\frac{I_{\text {observed }}}{I_{\text {cloud }}}=\left(\frac{d}{r_{\text {LMC }}}\right)^{2} \cdot I_{\text {observed }}$ is the stellar continuum intensity at $1000 \AA$ corrected for the extinction using the model of Cardelli et al. (1989), $I_{\text {cloud }}$ the intensity at the location of the cloud derived from our calculation, $r_{\mathrm{LMC}}$ is the distance to the LMC $(\approx 50 \mathrm{kpc})$ and $d$ is the distance between the cloud and the background star. We find that the cloud cannot be any closer than 120 pc from Sk-67D05. This alone suggests that the molecular component we are looking at is probably not associated with the star formation region. The details of the MCs $\mathrm{H}_{2}$ investigations ( $J$ levels, temperatures, radiation fields and $b$-values) in each sightline are shown in Table 9.

The derived values for the $\mathrm{HD} / \mathrm{H}_{2}$ ratio and the $\mathrm{CO} / \mathrm{H}_{2}$ ratios are respectively $1.5 \pm 0.5 \mathrm{ppm}$ and $2.7 \pm 0.7 \mathrm{ppm}$. These 
Table 9. Logarithmic column densities of molecular and atomic species detected in the $\mathrm{MCs}^{a}$.

\begin{tabular}{|c|c|c|c|c|c|}
\hline & Sk $-67 \mathrm{D} 05$ & $\mathrm{Sk}-68 \mathrm{D} 135$ & Sk-69D246 & AV 95 & Sk 159 \\
\hline $\mathrm{H}_{2} J=0$ & $19.28_{-0.06}^{+0.05}$ & $19.46_{-0.06}^{+0.10}$ & $19.40_{-0.04}^{+0.05}$ & $19.11_{-0.04}^{+0.04}$ & $18.85_{-0.08}^{+0.06}$ \\
\hline $\mathrm{H}_{2} J=1$ & $18.93_{-0.09}^{+0.04}$ & $19.61_{-0.04}^{+0.06}$ & $19.30_{-0.04}^{+0.04}$ & $19.11_{-0.04}^{+0.04}$ & $18.95_{-0.08}^{+0.06}$ \\
\hline $\mathrm{H}_{2} J=2$ & $15.60_{-0.30}^{+0.30}$ & $18.48_{-0.12}^{+0.12}$ & $17.70_{-0.50}^{+0.17}$ & $17.81_{-0.05}^{+0.05}$ & $17.48_{-0.10}^{+0.30}$ \\
\hline $\mathrm{H}_{2} J=3$ & $15.25_{-0.15}^{+0.25}$ & $18.23_{-0.08}^{+0.07}$ & $17.70_{-0.10}^{+0.20}$ & $17.54_{-0.07}^{+0.06}$ & $17.03_{-1.00}^{+1.30}$ \\
\hline $\mathrm{H}_{2} J=4$ & $14.48_{-0.06}^{+0.04}$ & $17.48_{-0.05}^{+0.12}$ & $15.49_{-0.17}^{+0.22}$ & $16.54_{-0.16}^{+0.12}$ & $13.81_{-0.40}^{+1.12}$ \\
\hline $\mathrm{H}_{2} J=5$ & $\ldots$ & $16.30_{-0.07}^{+0.40}$ & $15.17_{-0.09}^{+0.45}$ & $15.48_{-0.13}^{+0.30}$ & $\ldots$ \\
\hline $\mathrm{H}_{2} J=6$ & $\ldots$ & $14.54_{-0.15}^{+0.20}$ & $13.90_{-0.12}^{+0.14}$ & $\ldots$ & $\ldots$ \\
\hline Total & $19.44 \pm 0.05$ & $19.87 \pm 0.05$ & $19.66 \pm 0.04$ & $19.43 \pm 0.04$ & $19.19 \pm 0.06$ \\
\hline$T_{01}(\mathrm{~K})$ & 57 & 92 & 70 & 78 & 78 \\
\hline$b_{\text {Dopp }}\left(\mathrm{km} \mathrm{s}^{-1}\right)$ & 5.1 & 4.4 & $3.6 ; 6.4^{b}$ & 0.8 & $1.0 ; 1.3^{b}$ \\
\hline$N\left(\mathrm{H}_{\text {total }}\right)$ & 21.49 & 21.50 & 21.32 & 21.00 & $\ldots$ \\
\hline HD $J=0^{c}$ & $13.62_{-0.12}^{+0.09}$ & $14.15_{-0.15}^{+0.11}$ & $13.62_{-0.08}^{+0.07}$ & $13.82_{-0.18}^{+0.96}$ & $13.85_{-0.14}^{+0.11}$ \\
\hline $\mathrm{CO}^{\mathrm{c}}$ & $13.88_{-0.09}^{+0.08}$ & $13.77_{-0.28}^{+0.20}$ & $13.57_{-0.09}^{+0.08}$ & $<13.23$ & $<13.34$ \\
\hline $\mathrm{ClI}$ & $13.87_{-0.13}^{+0.12}$ & $13.59_{-0.20}^{+0.18}$ & $13.46_{-0.22}^{+0.24}$ & $13.00_{-0.30}^{+0.18}$ & $<13.32$ \\
\hline $\mathrm{Cl}$ II & $<13.78$ & $\ldots$ & $13.73_{-0.05}^{+0.14}$ & $<14.00$ & $\cdots$ \\
\hline $\mathrm{Fe}$ II & $14.84_{-0.08}^{+0.20}$ & $15.77_{-0.30}^{+0.18}$ & $15.26_{-0.35}^{+0.11}$ & $15.22_{-0.50}^{+0.50}$ & $14.93_{-0.50}^{+0.50}$ \\
\hline$\chi^{d}$ & 3 & 3000 & 20 & 200 & 4 \\
\hline distance $(\mathrm{pc})$ & 120 & 5 & 28 & 3 & 50 \\
\hline$[\mathrm{Cl}]$ & $>4.64$ & 4.09 & 4.60 & 4.00 & $\ldots$ \\
\hline$\delta(\mathrm{Cl})^{e}$ & $>-0.12$ & -0.67 & -0.16 & $>-0.70$ & $\cdots$ \\
\hline $\mathrm{Cl} \mathrm{I} / \mathrm{H}_{2}$ (total) (ppm) & $2.7 \pm 0.9$ & $0.5 \pm 0.3$ & $0.6 \pm 0.4$ & $0.4 \pm 0.3$ & $<1.3$ \\
\hline $\mathrm{Cl} \mathrm{I} / \mathrm{H}_{2}(\mathrm{~J}=0,1)(\mathrm{ppm})$ & $2.7 \pm 0.9$ & $0.6 \pm 0.3$ & $0.6 \pm 0.4$ & $0.4 \pm 0.3$ & $<1.3$ \\
\hline $\mathrm{HD} / \mathrm{H}_{2}$ (total) (ppm) & $1.5 \pm 0.5$ & $1.9 \pm 0.8$ & $0.9 \pm 0.2$ & {$[2.5 \ldots 22.4]$} & $4.5 \pm 1.8$ \\
\hline $\mathrm{CO} / \mathrm{H}_{2}$ (total) (ppm) & $2.7 \pm 0.7$ & $0.8 \pm 0.7$ & $0.8 \pm 0.2$ & $<0.63$ & $<1.33$ \\
\hline
\end{tabular}

a All the errors quoted are $1 \sigma$. The upper limits are $3 \sigma$.

$b$ Two molecular components are detected towards Sk-69D246 and Sk 159.

$c$ Average between PF and AOD techniques.

$d$ The Interstellar Radiation Field (ISRF) at $1000 \AA$ is given in Draine units $\left(7 \times 10^{-17} \mathrm{erg} \mathrm{cm}^{-3} \AA^{-1}\right)$.

$e$ The depletion of Chlorine, $\delta(\mathrm{Cl})$, is the difference between the LMC and SMC values of 4.76 and 4.70, respectively (see Russell \& Dopita 1992) and the values we derived here.

values are comparable to the ones derived in the MW disk (Ferlet et al. 2000; Lacour et al. 2003 in prep). Several important questions are raised by these relatively large ratios. Firstly, the $\mathrm{CO}$ column density is directly influenced by the availability of $\mathrm{C}$ and $\mathrm{O}$ atoms in the gas phase; while the $\mathrm{C}$ and $\mathrm{O}$ atoms are each depleted by about 0.5 dex (Russell \& Dopita 1992), the $\mathrm{CO}$ seems not to be depleted at all. Secondly, the lower metallicity should also result in fewer dust grain surfaces and thus in suppressed $\mathrm{H}_{2}$ formation. But most of the $\mathrm{CO}$ lines appear near the core of the $\mathrm{H}_{2}$ absorption bands and it is usually assumed that $\mathrm{H}_{2}$ is an efficient photo-dissociation shield for $\mathrm{CO}$. The $\mathrm{CO} / \mathrm{H}_{2}$ ratio would then tend to decrease when the metallicity decreases. Thirdly, the relatively small $\mathrm{H}_{2}$ column we derive should result in a less efficient FUV-shield in this LMC cloud and a smaller $\mathrm{CO} / \mathrm{H}_{2}$ ratio whatever the dust content along the sightline.

We also detected neutral chlorine atoms which can only exist in the molecular component (see Sect. 4.5). We find a $\mathrm{Cl} \mathrm{I} / \mathrm{H}_{2}$ ratio of $2.74 \pm 0.9 \mathrm{ppm}$, a value at least twice as high as what has been found in the Galactic disk towards HD 192639 (Sonnentrucker et al. 2002) and HD 185418 (Sonnentrucker et al. 2003). Detailed analysis of the latter lines of sight indicated that the gas was made up of multiple neutral diffuse clouds. The fact that we derive a much higher ratio along a 
sightline where the metallicity is lower by 0.5 dex than the Galactic value, strongly suggests that denser molecular clumps are present towards Sk-67D05.

It is possible to get a crude estimate of the total H I column density in the LMC using the gas-to-dust ratio derived by Koornneef (1982) of $N(\mathrm{HI}) / E_{B-V}=2 \times 10^{22} \mathrm{mag}^{-1} \mathrm{~cm}^{-2}$. However, we must correct the $E_{B-V}$ for potential MW gas contamination. Thus we calculated the MW reddening assuming $N\left(\mathrm{H}_{2}\right) / E_{B-V}=5 \times 10^{20} \mathrm{mag}^{-1} \mathrm{~cm}^{-2}$ (Dufour 1982). We find that, towards $\mathrm{Sk}-67 \mathrm{D} 05$, the Galactic material represents only a small fraction of the total reddening of about $E_{B-V(\mathrm{MW})} \approx 0.01$, and thus a small fraction of the total $N(\mathrm{HI})$ of about $10^{19} \mathrm{~cm}^{-2}$. Consequently, we derive $N(\mathrm{HI})_{\text {total }} \approx N(\mathrm{HI})_{\mathrm{LMC}} \approx 3 \times 10^{21} \mathrm{~cm}^{-2}$. This $\mathrm{HI}$ column density is about 6 times as large as the one derived towards the same line of sight by Friedman et al. (2000). The difference is mainly due to the fact that Friedman et al. (2000) used the Galactic gas-to-dust ratio which is 4 times as small as the LMC value (Koornneef et al. 1982). In our case, where most of the $\mathrm{HI}$ originates in the LMC, this can lead to a large systematic error. Furthermore, we adopted the updated $E_{B-V}$ as quoted in Tumlinson et al. (2002) which is 1.5 times as large as the value used by Friedman et al. (2000).

It could be argued that NaI might be used as a fiducial for HI with greater accuracy than the gas-to-dust ratio. Indeed, investigations of the column density correlation $N(\mathrm{NaI})$ versus $N(\mathrm{HI})$ by Ferlet et al. (1985) in the MW have demonstrated that Na I roughly follows HI over more than three orders of magnitude. However, the abundance of sodium in the LMC gas varies between sightlines. In the detailed investigation of Welty et al. (1999) towards SN1987A, about 20 individual LMC components were found with $\log N(\mathrm{NaI}) / N\left(\mathrm{H}_{\text {total }}\right)$ ranging from $\approx-9.0$ to $-9.7 \mathrm{dex}$. Here, we assume that the ratios between $\mathrm{Na}$ I components are reasonably linked to the ratios between $\mathrm{H}$ I components and we derived $N(\mathrm{HI}) \approx 8 \times 10^{20} \mathrm{~cm}^{-2}$ within the absorber associated with the $\mathrm{H}_{2}$ component, leading to a molecular fraction $f\left(\mathrm{H}_{2}\right)=2 N\left(\mathrm{H}_{2}\right) /\left(N(\mathrm{HI})+2 N\left(\mathrm{H}_{2}\right)\right)$ of around $7 \%$ on average over the $\mathrm{Na}$ components. This result together with the finding that, on average, the $\mathrm{CO}$ population ratio is subthermally distributed suggests that some more diffuse gas is also present along the Sk-67D05 light path.

Finally, we measured the column density of Fe II and found a value consistent with the analysis by Friedman et al. (2000): $N(\mathrm{Fe}$ II $)=7.0_{-1.5}^{+3.0} \times 10^{14} \mathrm{~cm}^{-2}$. Note that this represents the total amount of Fe II as seen in the four LMC components. Although the profile fitting made use of the four components at the same time, interpretation of Fe II in each component is difficult because these are not resolved by FUSE. Fortunately, most of the Fe II lines used in the profile fitting are in the optically thin regime. Hence, the total Fe II we quote remains accurate. The observed abundance of $\mathrm{Fe}$ is $\log \left[N(\mathrm{Fe}\right.$ II $\left.) / N\left(\mathrm{H}_{\text {total }}\right)\right]+12=5.4$ where $N\left(\mathrm{H}_{\text {total }}\right)$ is the total amount of $\mathrm{H}$ atoms derived from the four absorbers using $\mathrm{NaI}$. When compared with the Fe abundance in the LMC (Russell \& Dopita 1992), our result implies a depletion of about 2 dex. Hence large Fe II depletion occurs towards Sk-67D05 although it is not clear if Fe II is depleted onto grains or in the ionized form. The fact that the $\mathrm{CO}$,
HD and ClI analyses strongly suggest the presence of dense molecular clumps could argue in favor of depletion onto grains but the present data do not allow us to rule out the possibility that Fe II might be predominantly ionized.

\section{2. $S k-68 D 135$}

Sk-68D135 is an ON9.7 star located in the North of the 30 Doradus region which is the largest H II region in the local group of galaxies (Fitzpatrick \& Savage 1984; Tumlinson et al. 2002). It is believed that this region requires a Lyman continuum radiation field equivalent to some $100 \mathrm{O} 5$ stars to maintain its ionization. The $\mathrm{H} \mathrm{I}$ data from Wayte (1990) at $8 \mathrm{~km} \mathrm{~s}^{-1}$ resolution reveal the presence of at least four absorbers along the sightline. Two of them arise in the MW disk and halo around +30 and $+90 \mathrm{~km} \mathrm{~s}^{-1}$ in heliocentric velocity, while the other two originate in the LMC at +210 and $+290 \mathrm{~km} \mathrm{~s}^{-1}$ in heliocentric velocity. Our data however indicate the presence of 5 groups of absorbers split into 17 components and centered at (1) $+30 \mathrm{~km} \mathrm{~s}^{-1}$; (2) $+80 \mathrm{~km} \mathrm{~s}^{-1}$; (3) $+160 \mathrm{~km} \mathrm{~s}^{-1}$; (4) $+220 \mathrm{~km} \mathrm{~s}^{-1}$; and (5) $+280 \mathrm{~km} \mathrm{~s}^{-1}$ (Table 7). The four components observed by Wayte (1990) can then be identified with group 1,2, 4, and 5. We thus confirm that these components are in the foreground of Sk-68D135. We note also a LMC HVC located at $+320 \mathrm{~km} \mathrm{~s}^{-1}$ showing a large Ca II absorption relative to the corresponding $\mathrm{Na}$ I absorption and probably indicating shocked material. Our profile fittings of the Ca II and Na I VLT data are shown in Fig. 4.

$\mathrm{H}_{2}$ arising in the MW is detected in the FUSE spectrum and we identify this molecular absorber with the atomic absorber seen in $\mathrm{NaI}$ at $41 \mathrm{~km} \mathrm{~s}^{-1}$. Not only is this absorber the strongest in $\mathrm{NaI}$ but it also exhibits a Na I/Ca II ratio of the order of 0.3 dex, typical of cold components (Welty et al. 1999). Note that adopting the complete Na I velocity structure does not improve the fit. This MW molecular component is a much larger than the one observed towards Sk-67D05. A summary of the $\mathrm{MW} \mathrm{H}_{2}$ analysis is given in Table 8 . We obtain $\log N\left(\mathrm{H}_{2}\right)=18.29 \pm 0.04 \mathrm{dex}, T_{01} \approx 103 \mathrm{~K}$, and $T_{23} \approx 102 \mathrm{~K}$.

The investigation of the velocity structure for the molecular species observed in the LMC is a little more difficult since there are four LMC components blended within $20 \mathrm{~km} \mathrm{~s}^{-1}$. The profile fitting shows that the best solution is consistent with a dominant absorber at $+269 \mathrm{~km} \mathrm{~s}^{-1}$ along with marginal contribution from an absorber at $+277 \mathrm{~km} \mathrm{~s}^{-1}$ (less than $1 \%$ ). We note that the absorber at $+269 \mathrm{~km} \mathrm{~s}^{-1}$ has the largest $\mathrm{NaI} / \mathrm{Ca}$ II ratio of the four components and is the most likely to harbor dust grains and molecules. After profile fitting analysis assuming a single absorber, we confirm most of the $\mathrm{H}_{2}$ measurements performed in the survey of $\mathrm{H}_{2}$ Magellanic Clouds sightlines by Tumlinson et al. (2002). However, for the higher levels, our estimates of the mean column density are somewhat different and lead us to different excitation temperatures. We find $\log N\left(\mathrm{H}_{2}\right)=19.87 \pm$ $0.05 \mathrm{dex}, T_{01} \approx 92 \mathrm{~K}$ and $T_{23} \approx 251 \mathrm{~K}$. Calculation of the incident radiation field using the same assumptions as for Sk-67D05 gives $U_{\lambda} \approx 2.2 \times 10^{-13} \mathrm{erg} \mathrm{cm}^{-3} \AA^{-1}$ which is several thousand times as large as the Galactic radiation field. This is a tremendously high value suggesting that the cloud 


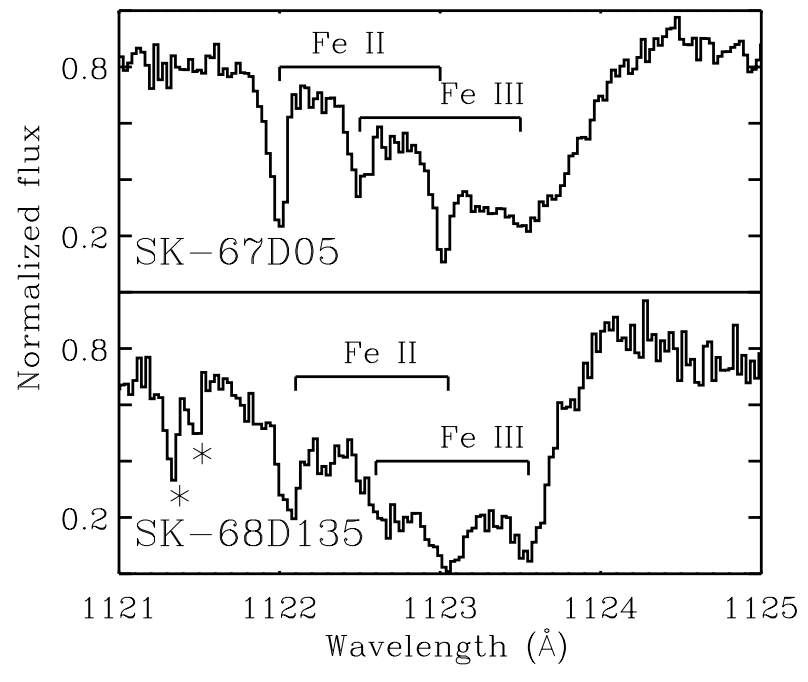

Fig. 8. The Fe II and Fe III absorption features arising in the MW and in the LMC towards Sk-68D135 (bottom panel) and Sk-67D05 (upper panel). Although the LMC Fe III line is barely seen towards $\mathrm{Sk}-67 \mathrm{D} 05$, we can see a strong detection towards Sk-68D135 consistent with the intense radiation field.

is located close to the star. If we assume that the background star is the only source irradiating the cloud, we find that the cloud is located at $5 \mathrm{pc}$ from the star. A likely scenario involves a larger molecular cloud being disrupted around the star $\mathrm{Sk}-68 \mathrm{D} 135$. We note that the incoming radiation field we derive is similar to the ambient radiation field estimated in the 30 Dor region using CO line emission (Bolatto et al. 1999 derived $\chi \approx 3000$ ). Another hint for a large radiation field comes from the observation of the strong Fe III lines at the LMC velocity. Figure 8 compares this line to the one observed towards Sk-67D05. Unfortunately, because the line is heavily saturated it was not possible to give a quantitative measurement for the $\mathrm{Fe}$ III/Fe II ratio. We note finally that this intense radiation field suggests that the cloud is embedded within the 30 Dor region and, therefore, may be at any distance from the background star.

That the $\mathrm{CO}$ and HD molecules are detected in such a harsh environment is puzzling. We actually find a $\mathrm{CO} / \mathrm{H}_{2}$ ratio of $0.80 \mathrm{ppm}$ and a $\mathrm{HD} / \mathrm{H}_{2}$ ratio of $1.9 \mathrm{ppm}$, values more typical of translucent $\left(A_{\mathrm{V}}>1\right)$ Galactic sightlines (Rachford et al. 2002). Despite the low chlorine abundance in the LMC (depletion of 0.5 dex, see Russell \& Dopita 1992), we find that the $\mathrm{Cl} / \mathrm{H}_{2}$ ratio is about $0.5 \mathrm{ppm}$ again, comparable to the Galactic measurements. These results, as for Sk-67D05, strongly suggest that higher density molecular clumps exist toward Sk-68D135 too.

Using the LMC gas-to-dust ratio (see Sect. 4.1), we find that the LMC HI column density can be as high as $5 \times$ $10^{21} \mathrm{~cm}^{-2}$. To derive the fraction of molecular absorbers we assumed that the $\mathrm{NaI}$ ratios between components translate into H I which would lead to a H I column density of about $3 \times$ $10^{21} \mathrm{~cm}^{-2}$ and a molecular fraction of $1 \%$. Models, however, show that such a low molecular fraction cannot be reconciled with the detected amount of HD molecules. The photodissociation time scale for the HD molecule is given by the photodissociation rate $\left(2.6 \times 10^{-11} \mathrm{~s}^{-1}\right.$ in the Galaxy, see Le Petit et al. 2002), $T_{\text {phot }} \approx 1500$ years. This time scale should be smaller in the LMC where more photons are available. When compared to the age of the star, $T_{\text {star }} \approx 10^{6}$ years, this time-scale is ridiculously small and suggests that HD is locked in a dense phase where the molecules are efficiently self-shielded and where the formation routes are enhanced.

As a matter of fact, because of the location of the cloud within the 30 Dor region, and if the cloud is a remnant of a denser molecular knot, we expect the survival of $\mathrm{CO}$, HD and other molecules in the core while the envelope, and all the $\mathrm{H}_{2}$ within it, is being swept away by shocks or stellar winds or destroyed by photons (thus reducing the measured integrated molecular fraction), as is observed. We note also that strong shocks are in action in 30 Dor and some of the dust grains might be destroyed, releasing in the gas phase many metals. The presence of a shock along the sightline to $\mathrm{Sk}-68 \mathrm{D} 135$ is reinforced by the detection of the LMC HVC component at $+308 \mathrm{~km} \mathrm{~s}^{-1}$ with an extremely low $\mathrm{Na}$ I/Ca II ratio of $-0.5 \mathrm{dex}$.

\section{3. $S k-69 D 246$}

The sightline towards Sk-69D246 has been extensively studied by Bluhm \& de Boer (2001). Sk-69D246 is a WN6 star located near R136, at the edge of the 30 Dor complex. IUE absorption lines of S II, and MgI as well as FUSE Fe II absorption lines show a velocity structure typical of most of the LMC sightlines (Danforth et al. 2002). We find three MW components around $0 \mathrm{~km} \mathrm{~s}^{-1}$, a disk cloud at $+40 \mathrm{~km} \mathrm{~s}^{-1}$, one IVC at $+80 \mathrm{~km} \mathrm{~s}^{-1}$, one $\mathrm{HVC}$ at $+130 \mathrm{~km} \mathrm{~s}^{-1}$, and four LMC components at $+234 \mathrm{~km} \mathrm{~s}^{-1},+260 \mathrm{~km} \mathrm{~s}^{-1},+280 \mathrm{~km} \mathrm{~s}^{-1}$, and $+290 \mathrm{~km} \mathrm{~s}^{-1}$. Our high resolution VLT data of Na I and Ca II make it possible to break the degeneracy associated with blended components not seen in IUE (Fig. 5). We are indeed able to detect 18 components as described in Table 7.

The MW molecular component is associated with the component at $25 \mathrm{~km} \mathrm{~s}^{-1}$ (as seen in $\mathrm{NaI}$ ) and has the same properties as the one seen towards Sk-68D135 except for a slightly larger total $\mathrm{H}_{2}$ column density. It is likely that both sightlines cross the same Galactic molecular cloud, and the comparison could set interesting limits on the variation scale across this absorber (see Sect. 5.4). We find $\log N\left(\mathrm{H}_{2}\right)=18.73 \pm 0.04 \mathrm{dex}$, and calculations of the Boltzmann temperatures yield $T_{01} \approx$ $83 \mathrm{~K}$ and $T_{23} \approx 179 \mathrm{~K}$.

The column densities for $J=0$ to $J=6$ in the LMC molecular component at $+279 \mathrm{~km} \mathrm{~s}^{-1}$ were derived by profile fitting and we noted that our fits improved noticeably when we added a second molecular component at $+285 \mathrm{~km} \mathrm{~s}^{-1}$ in heliocentric velocity. FUSE cannot fully resolve the two components, but we point out that a similar velocity structure was derived by Bluhm \& de Boer (2001). Furthermore, Cl I STIS data clearly confirm the presence of two molecular absorbers as shown in Fig. 5. For the total $\mathrm{LMC} \mathrm{H}_{2}$ column densities we find $\log N\left(\mathrm{H}_{2}\right)=$ $19.66 \pm 0.04 \mathrm{dex}, T_{01} \approx 70 \mathrm{~K}$ and $T_{23} \approx 352 \mathrm{~K}$. The balance of the $J=0,2$ and 4 levels yields (see Sect. 4.1 for details) $U_{\lambda} \approx 1.6 \times 10^{-15} \mathrm{erg} \mathrm{cm}^{-3} \AA^{-1}$. Assuming that the high $J$ levels are mainly populated by UV-pumping and assuming that 
the star is the principal source of UV photons, the clouds cannot be closer than $28 \mathrm{pc}$ from the background star Sk -69D246 and are possibly embedded in the 30 Dor region.

$\mathrm{CO}, \mathrm{HD}$ and $\mathrm{Cl} \mathrm{I}$ are also detected in the LMC molecular components in relatively large amounts. The $\mathrm{CO} / \mathrm{H}_{2}$ ratio is of the order of $0.8 \mathrm{ppm}$, the $\mathrm{HD} / \mathrm{H}_{2}$ ratio is $0.9 \mathrm{ppm}$ and the $\mathrm{Cl} \mathrm{I} / \mathrm{H}_{2}$ ratio is $0.6 \mathrm{ppm}$. A priori, these values are unexpected in the LMC, especially in 30 Dor unless, as for the two other stars, dense clumps exist and shield these molecules from the high UV radiation field.

We derive the HI column density associated with the LMC molecular components following the two assumptions described in Sect. 4.1: $N(\mathrm{HI})=2 \times 10^{21} \mathrm{~cm}^{-2}$, two times as large as the one quoted in Bluhm \& de Boer (2001) who used a direct relation between S II and H I. Because the two S II components are not resolved in the IUE data and because the S II lines are saturated, the analysis of S II data is difficult and does not allow one to take into account saturation effects in the core of the absorption lines. Thus, the HI column density may have been underestimated in the work of Bluhm \& de Boer (2001). The iron abundance is 5.96 leading to a depletion of 1.3 dex compared to the assumed LMC abundance (Russell \& Dopita 1992). Iron seems therefore to be strongly depleted. At this point, we cannot decide whether iron was preferentially depleted onto grains or is strongly ionized by the high ambient radiation field.

Owing to the relatively high signal-to-noise of the FUSE spectrum, the LMC Cl II absorption line is clearly detected at $1071 \AA$. At the same time, higher resolution STIS data of the Cl I line at $1347 \AA$ are also available. We have seen that Cl II is expected in the diffuse ISM where the hydrogen is atomic while $\mathrm{ClI}$ is associated with the molecular ISM. Adopting the Jura \& York (1978) model of a single cloud made of a skin, mainly atomic, and a core, mainly molecular, one can estimate the column density $N_{1}(\mathrm{H})$ of $\mathrm{HI}$ atoms coinciding with the optically thick $\mathrm{H}_{2}$ gas as follows: $N_{1}(\mathrm{H})=N\left(\mathrm{H}_{\text {total }}\right)$ $\left(f(\mathrm{Cl} \mathrm{I})-f\left(\mathrm{H}_{2}\right)\right)$ where $f(\mathrm{Cl} \mathrm{I})$ is the fraction of neutrals $(\mathrm{Cl} \mathrm{I})$ among the chlorine atoms, $f\left(\mathrm{H}_{2}\right)$ is the integrated molecular fraction along the sightline, $N\left(\mathrm{H}_{\text {total }}\right)$ is the integrated total column of hydrogen (atomic and molecular). We estimate that the molecular fraction inside the core of the molecular component, assuming similar physical properties and chlorine depletion, is about $12 \%$. Note that this is a strong lower limit since some of the diffuse H I may arise in separate pure atomic components and thus $N_{1}(\mathrm{H})$ may be overestimated.

The LMC C I, C I*, and C I** lines are also detected towards $\mathrm{Sk}-69 \mathrm{D} 246$, as shown in Fig. 9. Although many absorption line multiplets are present in the FUSE range, it is difficult to isolate unblended lines. We therefore employed the STIS data around $1275 \AA$ to perform the analysis as shown in Fig. 7, when possible. These lines were assumed to arise only in the molecular components and were fitted along with the LMC molecular hydrogen (same velocity structure, same $b$-values). The profilefitting analysis yields $\log N(\mathrm{C} \mathrm{I})=13.85_{-0.06}^{+0.06}, \log N\left(\mathrm{CI}^{*}\right)=$ $13.72_{-0.10}^{+0.10}$, and $\log N\left(\mathrm{CI}^{* *}\right)=13.44_{-0.06}^{+0.05}$ (see Table 6). The total fractional abundances of carbon in the excited states are $f_{1}=$ $N\left(\mathrm{C} \mathrm{I}^{*}\right) / N\left(\mathrm{CI}_{\text {total }}\right)=0.43 \pm 0.08$ and $f_{2}=N\left(\mathrm{C} \mathrm{I}^{* *}\right) / N\left(\mathrm{C} \mathrm{I}_{\text {total }}\right)=$ $0.16 \pm 0.02$. Assuming an average radiation field of ten times

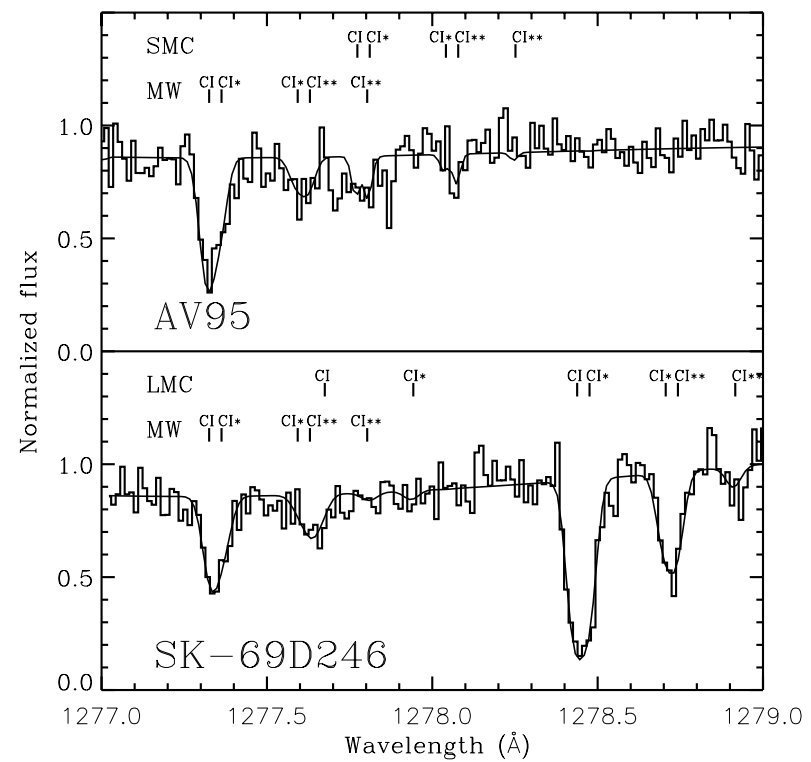

Fig. 9. Profile fitting of the carbon lines detected in the STIS data towards AV 95 and Sk-69D246.

the Galactic radiation field, we obtain $\log (P / k)=\log \left(n_{\mathrm{H}} T\right)$ between 4.3 and 4.6 (Jenkins \& Shaya 1979). If we assume that the carbon atoms are in the cold LMC molecular component at $72 \mathrm{~K}$, then we can expect the density $n_{\mathrm{H}}$ to be roughly between 300 and $600 \mathrm{~cm}^{-3}$. This should be considered a preliminary estimate since, among other simplifications, we have not taken into account the multiplicity of the sightline.

Additional evidence for the existence of a dense component comes from the detection of the $\lambda 4227 \AA \mathrm{Ca}$ I line at the LMC velocity. Even if the velocity structure of the line is buried within the noise of the spectrum, we can safely derive the total column density since the line is optically thin. We find $N(\mathrm{Ca} \mathrm{I})=1.5_{-0.2}^{+0.2} 10^{10} \mathrm{~cm}^{-2}$. If we assume ionization equilibrium, the $\mathrm{Ca} / \mathrm{CaII}$ ratio is a function of $n_{\mathrm{e}}$, the radiation field, and the temperature via the recombination coefficient $\alpha$ and the photoionization rate $\Gamma$ (see Sect. 4.6). Using $\alpha=5.58 \times 10^{-12} \mathrm{~cm}^{3} \mathrm{~s}^{-1}$ at $T \approx 100 \mathrm{~K}$ and $\Gamma=37 \times 10^{-11} \mathrm{~s}^{-1}$ (see Péquignot \& Aldrovandri 1986) multiplied by 20 to account for the larger radiation field, we find $n_{\mathrm{e}}$ ranging from 0.1 to $1 \mathrm{~cm}^{-3}$. A higher value is obtained if we add the Ca II component observed at $295 \mathrm{~km} \mathrm{~s}^{-1}$ which is probably too warm to be associated with the molecular absorbers. Compared to the previous density estimate, this electronic density leads to an ionization fraction of $1 \%$ at most.

\subsection{AV 95}

With the exception of the OVI lines studied by Howk et al. (2002), no investigation of the ISM towards AV 95 was found in the literature. Combining FUSE observations of Fe II with STIS observations of S II we detect 11 atomic components. Thus, this sightline is quite typical of SMC sightlines (Welty et al. 1997; Mallouris et al. 2001) showing a number of low-ionization Galactic gas clouds or complexes spread over $\approx 65 \mathrm{~km} \mathrm{~s}^{-1}$ (Fig. 10). Note that Fe II and S II ions trace the neutral component as well as the H II regions. The two species share the 


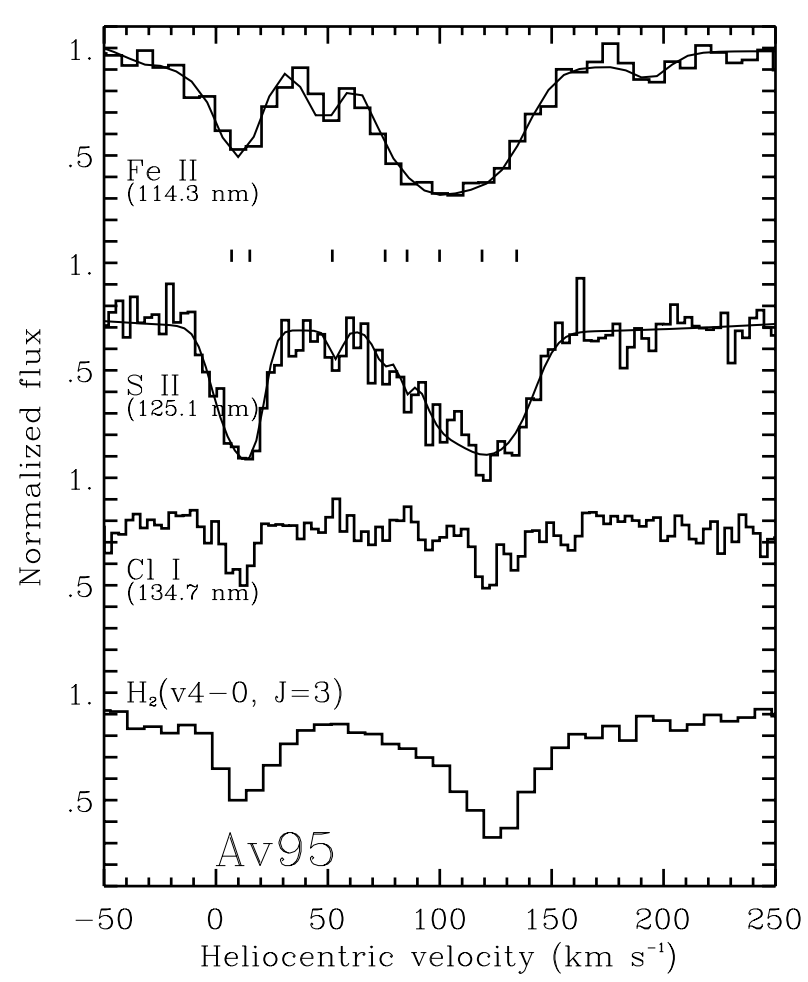

Fig. 10. Atomic and molecular velocity structures towards AV 95 as derived from STIS S II and FUSE Fe II and $\mathrm{H}_{2}$ data. Up to 11 atomic components are identified combining sulfur and iron absorbers. Note that with a resolution of $20 \mathrm{~km} \mathrm{~s}^{-1}$ many Fe II absorbers might well not be resolved and the Fe II column densities are uncertain. As to the molecular component, observations of Cl I with STIS at $\approx 6 \mathrm{~km} \mathrm{~s}^{-1}$ are consistent with a single molecular cloud.

same velocity structure but exhibit different ratios between components. Fe II in particular exhibits a few extra high velocity components at $+169 \mathrm{~km} \mathrm{~s}^{-1}$ and $+195 \mathrm{~km} \mathrm{~s}^{-1}$ (heliocentric velocities) that could correspond to shocked gas in which grain sputtering has released some of the refractory elements such as Fe into the SMC gas phase. The detailed velocity structure is summarized in Table 10.

The profile fitting of the FUSE data along with available STIS observations of $\mathrm{Cl}$ I at $\approx 6 \mathrm{~km} \mathrm{~s}^{-1}$ resolution is consistent with the presence of a single absorber in the MW and in the SMC with respective $b$-values of $1.5 \mathrm{~km} \mathrm{~s}^{-1}$ and $0.8 \mathrm{~km} \mathrm{~s}^{-1}$. For the MW component we derive $T_{01} \approx 66 \mathrm{~K}, T_{23} \approx 135 \mathrm{~K}$, $\log N\left(\mathrm{H}_{2}\right)=18.26 \pm 0.05$, and $N(\mathrm{Cl} \mathrm{I})=8.0 \times 10^{12} \mathrm{~cm}^{-2}$ which leads to a $\mathrm{Cl} / \mathrm{H}_{2}$ ratio of $0.4 \mathrm{ppm}$. Despite the large $\mathrm{H}_{2}$ column density we do not detect the HD molecule in this MW component.

The SMC CI, CI*, and CI** lines at $1276.48 \AA$, $1276.75 \AA$, and $1277.72 \AA$, available in the STIS spectra (Table 6), were first fitted along with SMC molecular hydrogen assuming common velocity structures and $b$-values. However, we found that these two assumptions were too strong and did not allow a proper fit of the carbon lines. Indeed, it appears that some of the carbon absorption lines arise in several components. Because our data did not allow us to reliably trace these more diffuse components, we performed a one-component fit that led to a total $b$-value of $5.7 \mathrm{~km} \mathrm{~s}^{-1}$ (about 6 times as large as the $\mathrm{SMC} \mathrm{H}_{2}$ adopted $b$-value). We note from the S II absorption lines detected in the FUSE range that there are many diffuse atomic components on this pathlength and some may well be cool enough to exhibit C I, C I*, and C I** lines. With these carbon lines, we inferred a range of possible $\mathrm{H}$ number densities for this component between 100 to $1000 \mathrm{~cm}^{-3}$. Due to the SMC blending it is not possible to derive the $\mathrm{HI}$ content directly. An alternative way is to estimate the total sulfur column density and calculate the total hydrogen column density assuming the SMC standard abundance $(\log N(\mathrm{~S} \mathrm{II}) / N(\mathrm{HI})=7.27$; see Russel \& Dopita 1992) and no depletion into the grains. The total Galactic sulfur column density is $N(\mathrm{~S})=2 \times 10^{15} \mathrm{~cm}^{-2}$ (the dominant ionization stage is $\mathrm{S}$ II) which leads to $N\left(\mathrm{H}_{\text {total }}\right)$ of the order of $10^{20} \mathrm{~cm}^{-2}$, and $f\left(\mathrm{H}_{2}\right)$ of only $3 \%$. The high $\mathrm{HD} / \mathrm{H}_{2}$ and $\mathrm{Cl} / \mathrm{H}_{2}$ ratios therefore suggest that denser clumps exist and are mixed with the more diffuse gas also present along the light path to AV 95 in the SMC.

The SMC molecular hydrogen is similar to that in the LMC with $T_{01} \approx 78 \mathrm{~K}, T_{23} \approx 245 \mathrm{~K}$, and $\log N\left(\mathrm{H}_{2}\right)=$ $19.43 \pm 0.04$. As above, we derive the H I column density using S II $\left(\log N(\mathrm{~S} \mathrm{II}) / N(\mathrm{HI})_{\mathrm{SMC}}=6.6\right.$; see Russel \& Dopita 1992), and find $N(\mathrm{HI}) \approx 10^{21} \mathrm{~cm}^{-2}$ and $f\left(\mathrm{H}_{2}\right) \approx 2.5 \%$. Again, some diffuse gas must lie along the sightline in the SMC gas too. From the population of the $J=0,2$ and 4 levels we derive a radiation field af $1.8 \times 10^{-14} \mathrm{erg} \mathrm{cm}^{-3} \AA^{-1}$ which is 250 times as large as the average Galactic radiation field. Hence, assuming that most of this incident flux comes from the background star, we obtain a lower limit on the distance between the star and the absorber of $3 \mathrm{pc}$.

The SMC CO molecule is barely detected in the FUSE spectra. We report a stringent $3 \sigma$ upper limit of $\log N(\mathrm{CO})<13.23$. This result is consistent with the fact that fewer oxygen atoms and fewer carbon atoms are available to form the $\mathrm{CO}$ molecule and that $\mathrm{CO}$ is more strongly photodissociated on this line of sight, the molecular clump density being probably lower (see our previous $\mathrm{C}$ I results).

The SMC ClI line is clearly detected towards this star in the STIS data (1347 $\AA$ ). The $\mathrm{Cl} \mathrm{I} / \mathrm{H}_{2}$ ratio is 0.4 ppm and translates into a depletion as much as 0.70 dex as shown in Table 9 , assuming $[\mathrm{Cl}]=4.70$ in the SMC (Russell \& Dopita 1992). However, we note that the possible presence of $\mathrm{ClII}$ might partly explain the low value. Indeed, the lower limit derived for the $\mathrm{Cl}$ II ion is consistent with no depletion of chlorine atoms at all. Another test of the depletion along the sightline is provided by the Fe II atoms. Using $\mathrm{H}$ I derived from S II, we find an iron abundance of $\approx 6.4$ instead of 6.84 as derived in the SMC by Russell \& Dopita (1992). The relatively low iron depletion, only 0.44 dex instead of 2 dex usually reported in the MW ISM (Savage \& Sembach 1996), can be due to a low dust content or can be the signature of shocks, past or present (as revealed by the two SMC HVCs at $+169 \mathrm{~km} \mathrm{~s}^{-1}$ and $+195 \mathrm{~km} \mathrm{~s}^{-1}$ ). Even though the depletion is uncertain, there are clear hints of a cold medium $\left(T_{01}=78 \mathrm{~K}\right)$ with a $b$-value as low as $0.8 \mathrm{~km} \mathrm{~s}^{-1}$ (as derived from the simultaneous fit of several species) so that we cannot rule out the presence of a dense core which might harbor a D-reservoir. A D-reservoir is defined as the inner region of a cloud where all of the deuterium atoms (hydrogen atoms) are in the form of $\mathrm{HD}\left(\mathrm{H}_{2}\right)$ molecules and where the $\mathrm{D} / \mathrm{H}$ ratio thus 
Table 10. S II and Fe II component parameters toward the SMC.

\begin{tabular}{|c|c|c|c|c|c|c|c|}
\hline Star & Comp & $\begin{array}{l}V_{\text {hel }} \\
\text { Fe II }\end{array}$ & $\begin{array}{c}N_{10} \\
10^{14} \mathrm{~cm}^{-2}\end{array}$ & $\begin{array}{c}b \\
\mathrm{~km} \mathrm{~s}^{-1}\end{array}$ & $\begin{array}{l}V_{\text {hel }} \\
\text { S II }\end{array}$ & $\begin{array}{c}N_{11} \\
10^{14} \mathrm{~cm}^{-2}\end{array}$ & $\begin{array}{c}b \\
\mathrm{~km} \mathrm{~s}^{-1}\end{array}$ \\
\hline \multirow[t]{11}{*}{ AV 95} & 1 & -15 & $>12.0^{a}$ & $<15.0^{a}$ & & & \\
\hline & 2 & 10 & 4.7 & 8.9 & 7 & 11.1 & 9.1 \\
\hline & 3 & & & & 15 & 11.3 & 5.9 \\
\hline & 4 & 48 & 2.4 & 4.7 & 52 & 31.0 & 0.3 \\
\hline & 5 & 75 & $>0.9^{a}$ & $<11 .^{a}$ & 75 & 1.0 & 4.4 \\
\hline & 6 & & & & 85 & 1.6 & 5.1 \\
\hline & 7 & 95 & 9.7 & 17.4 & 99 & 8.7 & 8.1 \\
\hline & 8 & 123 & 7.1 & 16.8 & 119 & $>20.0^{a}$ & $<11.0^{a}$ \\
\hline & 9 & & & & 134 & 9.0 & 7.7 \\
\hline & 10 & 169 & 0.9 & 28.6 & & & \\
\hline & 11 & 195 & $>0.6^{a}$ & $<21.0^{a}$ & & & \\
\hline \multirow[t]{3}{*}{ Sk 159} & 1 & -2 & 1.1 & 2.5 & & & \\
\hline & 2 & 23 & 8.1 & 16.9 & & & \\
\hline & 3 & 150 & 8.6 & $\ldots^{b}$ & & & \\
\hline
\end{tabular}

${ }^{a}$ Lower and upper limits quoted are $3 \sigma$.

${ }^{b}$ For this very saturated component the possible $b$-value ranges from 1 to $40 \mathrm{~km} \mathrm{~s}^{-1}$.

translates into $\mathrm{HD} / 2 \times \mathrm{H}_{2}$; these hypothetical places are of cosmological interest since they would allow accurate $\mathrm{D} / \mathrm{H}$ measurements throughout the MW disk (Ferlet et al. 2000). The predominance of diffuse gas, however, renders the study of this colder denser molecular gas more difficult.

Relatively large amounts of the HD molecule are detected in the SMC towards AV 95. Using both the AOD and PF techniques, we find $\log N(\mathrm{HD})=13.82_{-0.18}^{+0.96}$. The large error bar is due to the possible saturation of some of the HD lines detected in the spectra. Interestingly enough, $\mathrm{HD} / 2 \mathrm{H}_{2}$ ranges from $1.6 \mathrm{ppm}$ to $21 \mathrm{ppm}$ within $1 \sigma$. The latter value, if confirmed, would correspond to the first clear detection of a D-reservoir ever (Lacour et al. 2004). Moreover, the existence of a D-reservoir is not excluded by the range of densities derived, although crudely, through the $\mathrm{C}$ I excited states.

\subsection{Sk 159}

Sk 159 is located within Knot 1 (K1) which is rich in H II regions and is located in the foreground compared with the main body of the SMC (De Vaucouleurs \& Freeman 1972). This region is remarkable for the homogeneity of the stellar radial velocities with a typical dispersion of $5 \mathrm{~km} \mathrm{~s}^{-1}$. The investigation of this region based on Na I lines by Silvy (1996) showed that Sk 159 is located on the extreme edge of the $\mathrm{H}$ complex within the $\mathrm{K} 1$ region. The Na I data are consistent with the presence of a dominant SMC atomic absorber at $+144 \mathrm{~km} \mathrm{~s}^{-1}$ and a smaller one at $+149 \mathrm{~km} \mathrm{~s}^{-1}$. In the FUSE data no intermediate components and no SMC HVCs are detected in Fe II. The profile fitting in the FUSE range is consistent with a single atomic component near $+150 \mathrm{~km} \mathrm{~s}^{-1}$. However, high resolution $\mathrm{Na}$ I data obtained at the ESO $3.6 \mathrm{~m} / \mathrm{CES} / \mathrm{VLC}$ by Welty (private communication) clearly show two identical components separated

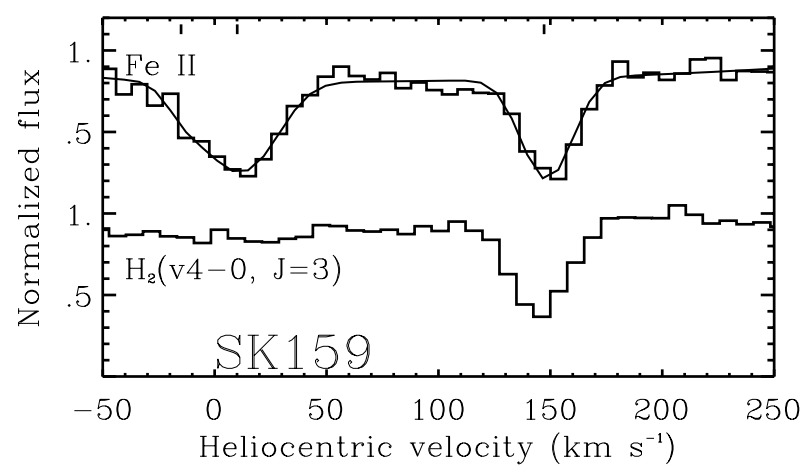

Fig. 11. Atomic and molecular velocity structures towards Sk 159. Based on the FUSE data itself, only one SMC aborber is revealed. However, higher resolution data, obtained at the 3.6 CES at ESO Chile, shows that there are at least 2 atomic absorbers present along the sightline at +144 and $+149 \mathrm{~km} \mathrm{~s}^{-1}$ (Silvy 1996).

by approximately $5 \mathrm{~km} \mathrm{~s}^{-1}$. The relative simplicity of this sightline is likely the double effect of the position of the star near the edge of the SMC and the weak foreground Galactic material. The H I radio data obtained by Wayte (1990) show two components at $+150 \mathrm{~km} \mathrm{~s}^{-1}$ and $+190 \mathrm{~km} \mathrm{~s}^{-1}$. The non-detection of the $\mathrm{H}$ component at $+190 \mathrm{~km} \mathrm{~s}^{-1}$ in absorption (Fig. 11) shows that most of the material seen in radio is located behind the star.

The MW molecular component is quite small with $\log$ $N\left(\mathrm{H}_{2}\right)=16.31 \pm 0.25$ and quite warm with $T_{01} \approx 153 \mathrm{~K}$. Hence, this component ressembles those observed in the Galactic halo by Richter et al. (1999).

The SMC $\mathrm{H}_{2}$ was fitted assuming a velocity structure similar to that of $\mathrm{NaI}$ (see Welty, private communication). We thus performed profile fitting of the FUSE data with this structure which led us to tentatively detect 2 molecular components: one at $+143 \mathrm{~km} \mathrm{~s}^{-1}$ and the other at $+152 \mathrm{~km} \mathrm{~s}^{-1}$. We 
find that the integrated properties are similar to the ones observed towards AV 95 with $T_{01} \approx 87 \mathrm{~K}, T_{23} \approx 204 \mathrm{~K}$, and $\log N\left(\mathrm{H}_{2}\right)=19.21 \pm 0.06$. Calculation of the incident radiation field gives $3.0 \mathrm{erg} \mathrm{cm}^{-3} \AA^{-1}$ which indicates that the clouds are at least $50 \mathrm{pc}$ from Sk 159. We report on a possible weak detection of the SMC CO molecule with $\log N(\mathrm{CO})<13.34$ ( $3 \sigma$ upper limit). On the contrary, the SMC HD molecule is clearly detected in the data with $\log N(\mathrm{HD})=13.85_{-0.14}^{+0.11}$ and $\mathrm{HD} / \mathrm{H}_{2}=4.5 \mathrm{ppm}$. Thus, we obtain a lower limit to the $\mathrm{D} / \mathrm{H}$ ratio of $2.3 \times 10^{-6}$ inside that component. The D-reservoir does not seem to have been reached. We would like to point out that this analysis is only based on FUSE data and a $b$-value of $2.6 \mathrm{~km} \mathrm{~s}^{-1}$, but higher resolution data might unravel colder components buried in the absorption lines and might lead to a higher HD column density.

\section{Discussion}

\subsection{General properties of the ISM in the MCs}

In their large survey of the diffuse molecular ISM in the MCs, Tumlinson et al. (2002) explored some of the global properties of this gas, comparing these properties to typical Galactic properties, and derived a reduced $\mathrm{H}_{2}$ formation rate, $R \approx$ $3 \times 10^{-18} \mathrm{~cm}^{3} \mathrm{~s}^{-1}$ (one third to one tenth the Galactic rate), as well as reduced molecular fractions $f\left(\mathrm{H}_{2}\right)$ of the order of $1 \%$ (one tenth the Galactic mean). These results were interpreted as the effect of suppressed $\mathrm{H}_{2}$ formation on grains and enhanced destruction by FUV photons. The total diffuse $\mathrm{H}_{2}$ masses were then found to be less than $2 \%$ of the H I mass, suggesting either a low molecular content and a high star formation efficiency or the presence of substantial mass in cold, dense clouds unseen in their survey. Although we have only 5 stars at our disposal, we can pursue the investigation of this "pseudo" diffuse molecular ISM of the MCs with a closer look at species not investigated previously.

In this work, we have investigated three lines of sight towards the Large Magellanic Clouds and reported measurements of $\mathrm{HD}, \mathrm{CO}, \mathrm{ClI}$ and $\mathrm{H}_{2}$. A summary of these measurements is given in Table 9. The average $\mathrm{HD} / \mathrm{H}_{2}$ ratio is $1.4 \pm 0.5 \mathrm{ppm}$, the average $\mathrm{CO} / \mathrm{H}_{2}$ ratio is $1.4 \pm 0.5 \mathrm{ppm}$ (we find $0.8 \mathrm{ppm}$ when the large value towards $\mathrm{Sk}-67 \mathrm{D} 05$ is excluded), and the average $\mathrm{Cl} / / \mathrm{H}_{2}$ ratio is $1.3 \pm 0.5 \mathrm{ppm}$ where the quoted errors are the standard deviations. These measurements lead us to two major conclusions. Firstly, despite the large variation of physical conditions between the sightlines (the incident radiation field varies by 3 orders of magnitude, the total extinction varies by a factor 3 and so do the total $\mathrm{H}$ column densities) the standard deviation of these ratios is only of the order of $30 \%$. Thus, it seems that each time the HD molecule is detected in the LMC ISM we are indeed sampling the same kind of isolated cloud, whose individual properties are not averaged along the pathlength, and within which the shielding is efficent enough to reduce the influence of the radiation field.

Secondly, these ratios are similar to those derived in the Galactic ISM. This is a bit surprising given the low total extinction towards these stars, the large incident radiation field as derived from the UV pumping, and the low abundance of carbon and chlorine atoms in the LMC. The sightline towards $\mathrm{Sk}-68 \mathrm{D} 135$ gives the most extreme example. We have shown (Sect. 4.2) that the cloud is embedded within the 30 Dor region where the radiation field is about 3000 times as large as the typical Galactic value. Contrarily to the expectations, this sight line exhibits the largest $\mathrm{CO} / \mathrm{H}_{2}$, the largest $\mathrm{Cl}$ I depletion, and the largest HD column density in the present sample.

Towards the SMC, we report two detections of the $\mathrm{HD}$ molecule, one detection of $\mathrm{Cl} \mathrm{I}$, and two upper limits on the $\mathrm{CO}$ molecules. The $\mathrm{Cl} / / \mathrm{H}_{2}$ ratio is $0.4 \mathrm{ppm}$ towards $\mathrm{AV} 95$, well within the range of typical Galactic translucent clouds. The two lower limits that we derived for the $\mathrm{CO} / \mathrm{H}_{2}$ ratio are consistent with the fact that the $\mathrm{CO}$ abundance is expected to be significantly smaller in the SMC (see Sect. 5.1). The measurement of the HD molecule towards AV 95 yields a surprisingly high $\mathrm{HD} / \mathrm{H}_{2}$ ratio of $21 \mathrm{ppm}$ (the largest value of the whole sample). Such a large amount of HD molecules is unexpected along diffuse sightlines towards the MCs and this observation strongly suggests that dense clumps are present and contribute significantly to the total column densities.

However, such measurements may suffer from unknown systematics errors and we would like to state that the profile fitting of STIS Cl I data that strongly constrains the molecular components only shows saturated absorption lines for which the column densities are driven by the $b$-value. Our simultaneous analysis of FUSE and STIS data combining several species, however, confirms the presence of an extremely narrow SMC component $\left(0.8 \mathrm{~km} \mathrm{~s}^{-1}\right.$ of total $b$-value $)$, adding weight to the suggestion that denser molecular gas is present.

Another interesting comparison of the MW ISM with the MCs ISM comes from the molecular gas-to-dust ratio. With the direct measurements of the Galactic $\mathrm{H}_{2}$ column densities towards the MCs we are able to correct for reddening from the foreground Galactic material (Dufour et al. 1982; see Sect. 4.2) and derive the intrinsic MCs reddening. Note that for the five sightlines the corrections were found to be small, which is consistent with the fact that our sample is biased towards low foreground Galactic column densities (see Sect. 2.1). Finally, we find that the $\mathrm{H}_{2} / E_{B-V}$ in the LMC ranges from 1.7 to $5.2 \times$ $10^{20} \mathrm{mag}^{-1} \mathrm{~cm}^{-2}$ in Sk-67D05 and Sk-69D246 respectively. In the SMC we derive $4.7 \times 10^{20} \mathrm{mag}^{-1} \mathrm{~cm}^{-2}$ for the AV 95 line of sight and $1.7 \times 10^{20} \mathrm{mag}^{-1} \mathrm{~cm}^{-2}$ for Sk 159 . Contrary to the conclusion drawn in Bluhm \& de Boer (2001) towards $\mathrm{Sk}-69 \mathrm{D} 246$, we find that these ratios compare quite well with the Galactic value from Dufour et al. (1982) of $\mathrm{H}_{2} / E_{B-V} \approx$ $5 \times 10^{20} \mathrm{mag}^{-1} \mathrm{~cm}^{-2}$ but our small sample does not allow us to make further quantitative investigations. We wish to point out that the LMC value for the $N(\mathrm{HI}) / E_{B-V}$ ratio as derived by Koornneef (1982) is $\approx 2 \times 10^{22} \mathrm{mag}^{-1} \mathrm{~cm}^{-2}$ and is several times as large as in the MW disk. This is consistent with smaller molecular fractions in the LMC and the fact that fewer dust grains (meaning less reddening) are sampled on average in any sightline in the LMC than in the MW.

Our investigation of the LMC and SMC ISM leads us to propose the existence of individual dense molecular structures where the translucent properties of the studied sightlines arise. This would explain the remarkable constancy of the ratios we derived despite the drastically different Galactic 
environments. Furthermore, we propose that these clouds should be dense enough to shield efficiently $\mathrm{CO}$ and HD despite enormous differences noted in the incident radiation fields. A preliminary estimate of the $\mathrm{H}$ number density using the study of atomic carbon fine structure lines observed with STIS towards Sk-69D246 and AV 95 (see Table 6) led to an estimate that $n_{\mathrm{H}}>$ few $100 \mathrm{~cm}^{-3}$.

Finally, our analysis indicates that, as for the MW, the MC sightlines exhibit a mixture of diffuse and denser molecular gas. The cold molecular gas in the MCs is however more readily detected in the MC gas probably due to a lower diffuse/dense gas ratio. We indeed observe one order of magnitude less $\mathrm{H}_{2}$ than towards typical Galactic translucent sightlines.

\subsection{Molecular clumps or filaments in the MCs}

It has been known for some time that the interstellar $\mathrm{H}$ I of the LMC represents a cooler mixture of phases than in the MW. The first direct evidence was reported by Dickey et al. (1994) in their ATCA 21-cm survey within the LMC; they established that a cold atomic phase is present in the MC ISM, at variance with the lack of cold phases outside the optical boundary of the MCs (Mebold et al. 1991). Moreover, they showed that the cold atomic phase is more abundant in the LMC ISM than within the MW. A few years earlier, observations of the low CO luminosity of the MCs (Cohen et al. 1988; Israel et al. 1991) were reported to be marginally consistent with the relatively large amount of atomic gas present $\left(\approx 2 \times 10^{9} M_{\odot}\right)$. The difference between the observations of cold atomic phases and cold molecular phases was soon interpreted to be an effect of the phase mixing and clumpiness of the medium (Lequeux et al. 1994).

However, to date only a few hints on a clumpy molecular phase are available. Recent ESO-SEST observations have revealed a new picture of the LMC molecular clouds with higher complexity than previously observed and molecular clumps as small as $25 \mathrm{pc}$ or less, the limitation being the spatial resolution of the instrument. The SEST survey towards the LMC complex N11 by Israel et al. (2003) allows the identification of a population of dense molecular clouds $\left(n_{\mathrm{H}_{2}} \approx 3000 \mathrm{~cm}^{-3}\right)$ with relatively warm temperature $(60 \mathrm{~K}-150 \mathrm{~K})$. This observation confirms the model proposed by Lequeux et al. (1994) in which the molecular complexes are seen as a collection of identical clumps of uniform density (between $10^{3}-10^{5} \mathrm{~cm}^{-3}$ ) immersed in a non-uniform interclump medium with density $n_{\mathrm{H}} \approx 100 \mathrm{~cm}^{-3}$.

The detection of cold atomic clouds has also been reported by Kobulnicky \& Dickey (1999) in absorption in the Magellanic Bridge, a 10-degree region of diffuse gas linking the main SMC body to an extended arm of the LMC. Shortly after, Lehner (2002) confirmed the existence of small molecular absorbers in the Magellanic Bridge using FUSE data. Typical densities for diffuse molecular absorbers in the MW disk (Rachford et al. 2002; Sonnentrucker et al. 2002, 2003) vary roughly in the range $1-100 \mathrm{~cm}^{-3}$; assuming that these densities hold for the Magellanic bridge, and given the low column density reported by Lehner (2002) $\left(\log N\left(\mathrm{H}_{2}\right)<15\right.$ dex $)$

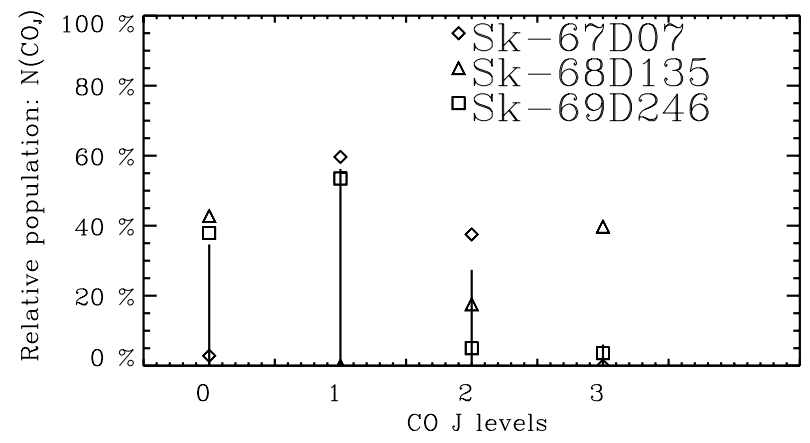

Fig. 12. The relative population distribution of the CO $J=1-3$ lines derived from the observations is compared to a theoretical Boltzmann distribution at $T_{\mathrm{ex}}(\mathrm{CO})=9 \mathrm{~K}$. The agreement is relatively good although the error bars on the individual measurements are of the order of $50 \%$ (not shown).

we compute the sizes of these clumps to be in the range 10-1000 Astronomical Units (AU).

Our present observations of molecular clumps in the MCs are consistent with Israel et al. (2003): with typical $\log N\left(\mathrm{H}_{2}\right) \approx$ 19 dex and density $>100 \mathrm{~cm}^{-3}$, we derive sizes of $10^{4} \mathrm{AU}$ or $1 / 10 \mathrm{pc}$ (well below the $25 \mathrm{pc}$ reported by Israel et al. 2003).

The detection of CO molecules in our LMC sightlines is strong evidence for even denser clumps, for it is usually assumed that densities ranging from 1000 to $100000 \mathrm{~cm}^{-3}$ are necessary to fit the observations (Lequeux et al. 1994). Recent investigations of the $\mathrm{H}_{3}^{+}$abundances led Cecchi-Pestellini \& Dalgarno (2000) to the same conclusions for the Galactic star Cygnus OB2 No. 12. Our observations also favor as well the existence of dense clumps embedded in a tenuous interclump medium. Not only do we find that the $\mathrm{CO} / \mathrm{H}_{2}$ ratio is relatively high, indicative of denser gas, but also that the investigation of the rotational levels of the $\mathrm{CO}$ molecules in the LMC targets leads to $\left\langle T_{\mathrm{ex}}(\mathrm{CO})\right\rangle \approx 9 \mathrm{~K}$ typical of subthermal excitation occurring in cold clouds (see Fig. 12).

In fact, if we adopt the model of clumpy diffuse ISM derived by Cecchi-Pestellini \& Dalgarno (2002) we find densities of the order of $10^{4} \mathrm{~cm}^{-3}$. At face value, this leads to extremely small clumps with sizes of $10^{3} \mathrm{AU}, 10^{2} \mathrm{AU}$, and smaller (the solar system is about $150 \mathrm{AU}$ ). However, several objections can be raised against the existence of such small molecular blobs. First, we can compare the probability for such an absorber to cross the light path to a MCs bright star with the actual covering factor. Assuming that the total molecular phase hidden in these blobs is of the order of $10^{7} M_{\odot}\left(10^{9} M_{\odot}\right.$ in the cold atomic phase $\times$ a molecular fraction of $1 \%$, see Dickey et al. 1999 and Tumlinson et al. 2002) over a $1 \mathrm{kpc} \times 1 \mathrm{kpc}$ sky area (the dimensions of the 30 Dor region), assuming that the blobs are randomly distributed in volume, then, knowing the radius $(0.05 \mathrm{pc})$ and the mass of each blob $\left(\approx 0.1 M_{\odot}\right.$ with $\left.n_{\mathrm{H}}=10^{4} \mathrm{~cm}^{-3}\right)$ we infer a crude covering factor of the order of $40 \%$. This is inconsistent with our observation that 3 LMC sightlines out of $55(\approx 5 \%)$ and 2 SMC sightlines out of $26(\approx 8 \%)$ are crossing these clumps (even taking into account a handful of possible misdetections; see Sect. 2.1). Note, however, that in the above picture it is assumed that most of the molecular mass resides in cold, dense clumps, which is not demonstrated. Second, if we 
assume that these blobs are roughly spherical then the dimensions we derive are ill-defined or should be considered only lower limits to the total radius. In effect, there is a larger probability to cross the outskirts than the core of such a clump and the frequency of core-crossing sightlines remains, by its nature, a hidden parameter. Anyhow, this latter scenario fails also to predict the observed similarities between sightlines (for instance, all the absorbers have total $\mathrm{H}_{2}$ column densities within a factor of two).

Filementary structures are, then, an interesting alternative. A small fraction of the molecular gas condensed in such structures could result in a relatively large covering factor if they are preferentially found near the stars (this is the case for all our targets except Sk-67D05). At the same time they provide a natural explanation for the approximately constant total $\mathrm{H}_{2}$ content since a deviation of 60 degrees from normal incidence upon a filament results in a mere factor two difference in the total crossing path.

The question of the origin of these small structures is outside the scope of this paper. Whether their formation is triggered by stellar formation processes or whether these clouds are small evaporating pieces of a parent body cannot be answered yet. Finally, we note that resilient molecular structures have to be present within star forming regions of the MCs and our conclusion follows the one by Dickey et al. (1994) who reported a clear correlation between the cold atomic phase and the star forming regions within the LMC.

\subsection{A possible lower limit for the $D / H$ ratio in the $S M C$ of $1.1 \times 10^{-5}$ ?}

Measurements of the atomic $\mathrm{D} / \mathrm{H}$ ratio have been performed in different astrophysical sites, in particular within Damped Lyman Alpha (DLAs) systems observed towards high redshift quasar absorbers. The DLAs have generally a few $\%$ solar metallicity and are the best candidates for investigating the primordial abundance of deuterium. However, these measurements are difficult to achieve and suffer from several systematics errors, one of which is the confusion due to the blending of between several H I absorbers along crowded lines of sight (Lemoine et al. 1999). At the present time, definitive conclusions cannot be reached although a trend towards $\mathrm{D} / \mathrm{H}_{\text {primordial }} \approx 3.5 \times 10^{-5}$ emerges (Kirkman et al. 2001; see for a review Lemoine et al. 1999). The $\mathrm{D} / \mathrm{H}$ ratio has also been thoroughly investigated in the Local ISM (see the review by Moos et al. 2002) with the FUSE satellite. These studies indicate that the deuterium abundance is approximately constant within $100 \mathrm{pc}$ at $1.5 \times 10^{-5}$ with a standard deviation of $20 \%$. This value is regarded as representative of the current $\mathrm{D} / \mathrm{H}$ ratio. Whether this abundance is representative of the current ISM or whether the LISM has peculiar abundances is still a question of debate. On rare occasions, the $\mathrm{D} / \mathrm{H}$ ratio has been measured in the MW along pathlengths greater than $1 \mathrm{kpc}$ and was consistently found to be smaller than the LISM value, around $0.8 \times 10^{-5}$ (Hoopes et al. 2003; Hébrard \& Moos 2003).

The determination of the deuterium abundance in the MCs is of great importance for the better understanding of the chemical evolution of the Universe through $\mathrm{D} / \mathrm{H}$ from the Big Bang Nucleosynthesis (BBN) to the current ISM. D/H in the SMC, with two tenths solar metallicity, would make it possible to have one measurement in the time range between the Damped Lyman Alpha systems (DLAs) formation and the current MW ISM. Unfortunately, because the deuterium lines appear next to the HI lines, the DI lines are usually severely blended with the strong Galactic H I lines at the SMC velocity.

Another way of investigating the $\mathrm{D} / \mathrm{H}$ ratio consists in studying the deuterated species present in the MC ISM such as $\mathrm{DCO}^{+}, \mathrm{DCN}, \mathrm{ND}_{3}$ or HD. However, apart from the case of the HD molecule, the models used to interpret the fractionation of the deuterium atoms are far from being reliable. For instance, recent observations of the ratio $\mathrm{NH}_{3} / \mathrm{ND}_{3}$ are in strong disagreement with the models, $\mathrm{ND}_{3}$ is found to be overabundant by a factor 10000 (van der Tak et al. 2002). Thus, although one $\mathrm{D} / \mathrm{H}$ measurement (around $1.5 \times 10^{-5}$ ) has been attempted via $\mathrm{DCO}^{+}$and $\mathrm{DCN}$ by Chin et al. (1996) in the SMC, the best probe for the deuterium abundance in the MCs still remains the $\mathrm{HD} / \mathrm{H}_{2}$ ratio provided there are molecular cores where the deuterium atoms are in the form of the HD molecules. In these so-called D-reservoirs $\mathrm{HD} / \mathrm{H}_{2}=2 \times \mathrm{D} / \mathrm{H}$.

At present, of all the extragalactic FUSE targets observed to date AV 95 is the most promising for that purpose. With a $\mathrm{HD} / \mathrm{H}_{2}$ ratio possibly as high as $2.2 \times 10^{-5}$, we can derive $\mathrm{D} / \mathrm{H}$ of the order of $1.1 \times 10^{-5}$, that is slightly smaller than the value found in the LISM but larger than the value measured towards the two extended MW sightlines HD 191877 and HD 195695 (Hoopes et al. 2003) with $\mathrm{D} / \mathrm{H}$ ratios of $0.78 \times 10^{-5}$ and $0.85 \times$ $10^{-5}$ respectively. For now, unfortunately, the analysis of the $\mathrm{HD} / \mathrm{H}_{2}$ ratio towards AV 95 with the existing FUSE data does not allow us to exclude a much more reduced value of $\mathrm{HD} / \mathrm{H}_{2}$ of $2 \times 10^{-6}$. In order to further investigate this interesting sightline higher resolution data are mandatory. Such high resolution data could be obtained in the optical by a tracer of $\mathrm{H}_{2}$ and HD (Lacour et al. 2004) such as CH at $4300 \AA$. This observational effort would not only lead to the first clear detection of a deuterium reservoir but also help to constrain cosmological models predicting the deuterium evolution as a function of metallicity.

\subsection{Molecular filaments detected in the MW}

LMC and SMC sightlines are especially well suited for the investigation of spatial variations in the ISM of our Galaxy (Ferlet 1999). Since the angular distance between MC background stars is much reduced compared with the MW stars, they can be used as probe of degree-scale structures in the MW. Two of the stars of our mini-survey are close enough to allow a test of these fine structures: Sk -68D135 and Sk-69D246 are within $30^{\prime}$ of each other. The same Na I component is detected around $+25 \mathrm{~km} \mathrm{~s}^{-1}$ with similar $b$-values for both stars.

At $1 \mathrm{kpc}, 30^{\prime}$ would correspond to a linear distance of $10 \mathrm{pc}$. We have seen that both clouds have kinetic temperatures around $100 \mathrm{~K}$, as deduced from $T_{01}$, with molecular hydrogen column densities around $\log N\left(\mathrm{H}_{2}\right) \approx 18$. Then, assuming that these absorbers are within the thick disk which 
extends to $\pm 250 \mathrm{pc}$ from the Galactic plane, the corresponding absorber scale is of the order of $2.5 \mathrm{pc}$ or smaller in the direction perpendicular to the sightline. It is reasonable to assume that we are indeed observing the same molecular structure.

In the case of Sk-69D246, the carbon lines detected in the STIS spectrum give an estimate of the density within the absorber from 60 to 600 atoms $\mathrm{cm}^{-3}$ (Table 6). Then, from the estimation of the total $\mathrm{H}_{2}$ column density, we have a rough estimate of the length of the medium crossed by the sightline in the direction of the sightline which is about $10^{3}$ to $10^{4} \mathrm{AU}$ or 0.1 to $0.01 \mathrm{pc}$.

It is clear from these calculations that the molecular structure observed is extremely filamentary with a ratio width/length in between $0.4 \%$ and $4 \%$.

Filamentary structures were observed for the first time in the Pleiades nebulosity (Osterbrock 1959) in optical data and later in radio via the polarization of the $\mathrm{H}$ I lines emitted in the Crab Nebula (Wright 1970). Today, such structures are commonly observed in the MW or even in the extragalactic ISM on scales of tens to hundreds of parsecs (Houlahan \& Scalo 1985; Kulkarni \& Heiles 1988; Curry 2000). Smaller scales have tentatively been identified in $\mathrm{H}$ I towards high-velocity pulsars and extragalactic radio sources (Frail et al. 1994; Faison et al. 1998) down to the AU scale. Confirmation is brought by recent spectroscopic investigations of the Galactic ISM gas (see Lauroesch et al. 2000; Richter et al. 2003; Rollinde et al. 2003) although it is not clear yet whether such structures, called TSAS for TinyScale Atomic Structures, are filamentary. Our present observations add weight, from the observational point of view, to the existence and the filamentary nature of the TSMS: Tiny-Scale Molecular Structures.

\section{Conclusions}

1. We have studied the sightlines towards three bright LMC stars: Sk-67D05, Sk-68D135, and Sk-69D246. The combination of ground-based observations (VLT) along with archival data from UV satellites (FUSE, STIS) makes it possible to investigate $\mathrm{H}_{2}, \mathrm{CO}, \mathrm{HD}, \mathrm{Cl}$ I, Cl II, C I, $\mathrm{CI}^{*}, \mathrm{CI}^{* *}$, Na I, Ca I, Ca II, Fe II, and S II. We also report on the investigation of two SMC sightlines with the help of FUSE and STIS data. With the exception of Na I, Ca I, and Ca II, we have obtained measurements or upper limits on all the species observed towards the LMC targets.

2. In the LMC we find $\left\langle\mathrm{HD} / \mathrm{H}_{2}\right\rangle=1.4 \mathrm{ppm}$, $\left\langle\mathrm{CO} / \mathrm{H}_{2}\right\rangle=1.4 \mathrm{ppm} \quad(0.8 \quad \mathrm{ppm}$ when excluding the large value obtained toward $\mathrm{Sk}-67 \mathrm{D} 05)$, and $\left\langle\mathrm{Cl} \mathrm{I} / \mathrm{H}_{2}\right\rangle=1.3 \mathrm{ppm}$. After correcting for the MW reddening we also derive $\left\langle N\left(\mathrm{H}_{2}\right) / E_{B-V}\right\rangle=3.5 \pm 1.8 \times$ $10^{20} \mathrm{mag}^{-1} \mathrm{~cm}^{-2}$. The analysis of the rotational levels of the $\mathrm{H}_{2}$ molecules gives a kinetic temperature of the crossed molecular gas of $\left\langle T_{01}\right\rangle \approx 70 \mathrm{~K}$. All these values are similar to values obtained in the MW disk and seem a bit surprising in the LMC where the radiation field is 5 to 3000 times as intense as in the MW disk and the metallicity lower by a factor 2. We suggest that these observations are consistent with the existence of cold, dense molecular structures embedded in the diffuse gas.
3. In the SMC, we find $\left\langle N\left(\mathrm{H}_{2}\right) / E_{B-V}\right\rangle=4.7$ and $1.7 \times$ $10^{20} \mathrm{mag}^{-1} \mathrm{~cm}^{-2}$ for AV 95 and Sk 159 respectively. The sightline towards AV 95 in particular shows an unexpectedly large $\mathrm{HD} / \mathrm{H}_{2}(21 \mathrm{ppm})$ ratio. We tentatively conclude that a D-reservoir is detected that can give access to a direct measurement of the $\mathrm{D} / \mathrm{H}$ in the SMC. However, more observations are needed, with better $S / N$ and higher resolution, to confirm our analysis.

4. The detailed analysis of the excited states of the carbon atoms gives an interesting determination of the $\mathrm{H}$ number density along the sightlines towards Sk-69D246 and AV 95. For both stars we find that the density is greater than $10^{2} \mathrm{~cm}^{-3}$ in the LMC molecular components. This leads to clump (or filament) sizes of the order of $0.1 \mathrm{pc}$. We note that there are hints for an even denser medium, and for sizes of the order of $10^{2} \mathrm{AU}(\approx 0.001 \mathrm{pc})$. We reach the same conclusions for the Galactic molecular component sampled by the Sk-68D135 and the Sk-69D246 sightlines. We argue that this component probably corresponds to a sheet-like structure such as the ones recently inferred in the Galactic halo (Richter et al. 2003). More quantitative conclusions are to be given in the following paper based on the chemical model of Le Petit et al. (2002).

Acknowledgements. This work was partially done using the procedure Owens.f developed by M. Lemoine and the FUSE french team. It is our great pleasure to thank Eric Maurice for bringing to our attention the PhD thesis of Jocelyne Silvy, and D. Welty for sharing with us some of his VLT data towards Sk 159. We gratefully acknowledge the referee, K. S. de Boer, for his helpful comments.

\section{References}

Abgrall, H., Roueff, E., Launay, F., Roncin, J. Y., \& Subtil, J. L. 1993a, A\&AS, 101, 273

Abgrall, H., Roueff, E., Launay, F., Roncin, J. Y., \& Subtil, J. L. 1993b, A\&AS, 101, 323

Abgrall, H., Roueff, E., \& Drira, I. 2000, A\&AS, 141, 297

Abgrall, H., \& Roueff, E. 2003, in preparation

Ballester, P., Grosbol, P., Banse, K., et al. 2000, Proc. SPIE, 4010, 246

Bertin, P., Lallement, R., Ferlet, R., \& Vidal-Madjar, A. 1993, A\&A, 278,549

Bluhm, H., \& de Boer, K. S. 2001, A\&A, 379, 82

Boehringer, H., \& Hartquist, T. W. 1987, MNRAS, 228, 915

Bolatto, A. D., Jackson, J. M., \& Ingalls, J. G. 1999, ApJ, 513, 275

Cardelli, J. A., Clayton, G. C., \& Mathis, J. S. 1989, ApJ, 345, 245

Cecchi-Pestellini, C., \& Dalgarno, A. 2000, MNRAS, 313, L6

Cecchi-Pestellini, C., \& Dalgarno, A. 2002, MNRAS, 331, L31

Chin, Y.-N., Henkel, C., Millar, T. J., Whiteoak, J. B., \& Mauersberger, R. 1996, A\&A, 312, L33

Chu, Y.-H., Wakker, B., Mac Low, M.-M., \& Garcia-Segura, G. 1994, AJ, 108, 1696

Cohen, R. S., Dame, T. M., Garay, G., et al. 1988, ApJ, 331, L95

Curry, C. L. 2000, ApJ, 541, 831

Curry, C. L. 2002, ApJ, 576, 849

Danforth, C. W., Howk, J. C., Fullerton, A. W., Blair, W. P., \& Sembach, K. R. 2002, ApJS, 139, 81

de Boer, K. S., Koornneef, J., \& Savage, B. D. 1980, ApJ, 236, 769

Dekker, H., D'Odorico, S., Kaufer, A., Delabre, B., \& Kotzlowski, H. 2000, Proc. SPIE, 4008, 534 
Dickey, J. M., Mebold, U., Marx, M., et al. 1994, A\&A, 289, 357

Diplas, A., \& Savage, B. D. 1994, ApJ, 427, 274

de Vaucouleurs, G., \& Freeman, K. C. 1972, Vistas in Astronomy, 14, 163

Draine, B. T. 1978, ApJS, 36, 595

Dufour, R. J., Shields, G. A., \& Talbot, R. J. 1982, ApJ, 252, 461

Ehrenfreund, P., Cami, J., Jiménez-Vicente, J., et al. 2002, ApJ, 576, L117

Faison, M. D., Goss, W. M., Diamond, P. J., \& Taylor, G. B. 1998, AJ, 116, 2916

Ferlet, R., Vidal-Madjar, A., \& Gry, C. 1985, ApJ, 298, 838

Ferlet, R. 1999, A\&ARv, 9, 153

Ferlet, R., André, M., Hébrard, G., et al. 2000, ApJ, 538, L69

Fitzpatrick, E. L., \& Savage, B. D. 1984, ApJ, 279, 578

Frail, D. A., Weisberg, J. M., Cordes, J. M., \& Mathers, C. 1994, ApJ, 436, 144

Friedman, S. D., Howk, J. C., Andersson, B.-G., et al. 2000, ApJ, 538, L39

Garay, G., Johansson, L. E. B., Nyman, L.-Å., et al. 2002, A\&A, 389, 977

Garnett, D. R., Shields, G. A., Peimbert, M., et al. 1999, ApJ, 513, 168

Hébrard, G., Lemoine, M., Vidal-Madjar, A., et al. 2002, ApJS, 140, 103

Hébrard, G., \& Moos, H. W. 2003, ApJ, 599, 297

Herbig, G. H. 1995, ARA\&A, 33, 19

Hoopes, C. G., Sembach, K. R., Hébrard, G., Moos, H. W., \& Knauth, D. C. 2003, ApJ, 586, 1094

Houlahan, P., \& Scalo, J. M. 1985, BAAS, 17, 835

Howk, J. C., Savage, B. D., Sembach, K. R., \& Hoopes, C. G. 2002, ApJ, 572, 264

Israel, F. P. 1991, IAU Symp., 146, Dynamics of Galaxies and Their Molecular Cloud Distributions, 146, 13

Israel, F. P., de Graauw, T., Johansson, L. E. B., et al. 2003, A\&A, 401, 99

Jenkins, E. B., \& Shaya, E. J. 1979, ApJ, 231, 55

Jenkins, E. B., Savage, B. D., \& Spitzer, L. 1986, ApJ, 301, 355

Jenkins, E. B., \& Tripp, T. M. 2001, ApJS, 137, 297

Jura, M. 1974, ApJ, 190, L33

Jura, M., \& York, D. G. 1978, ApJ, 219, 861

Kimble, R. A., Woodgate, B. E., Bowers, C. W., et al. 1998, Proc. SPIE, 3356, 188

Kirkman, D., Tytler, D., O’Meara, J. M., et al. 2001, ApJ, 559, 23

Kobulnicky, H. A., \& Dickey, J. M. 1999, AJ, 117, 908

Koornneef, J. 1982, A\&A, 107, 247

Kulkarni, S. R., \& Heiles, C. 1988, Galactic and Extragalactic Radio Astronomy, 95

Lauroesch, J. T., Meyer, D. M., \& Blades, J. C. 2000, ApJ, 543, L43

Lacour, S., Le Petit, F., Sonnentrucker, P., et al. 2004, in preparation

Lehner, N. 2002, ApJ, 578, 126

Lehner, N., Gry, C., Sembach, K. R., et al. 2002, ApJS, 140, 81

Lemoine, M., Audouze, J., Ben Jaffel, L., et al. 1999, New Astron., 4, 231

Lemoine, M., Vidal-Madjar, A., Hébrard, G., et al. 2002, ApJS, 140, 67

Le Petit, F., Roueff, E., \& Le Bourlot, J. 2002, A\&A, 390, 369

Lequeux, J., Le Bourlot, J., Des Forets, G. P., et al. 1994, A\&A, 292, 371

Mallouris, C., Welty, D. E., York, D. G., et al. 2001, ApJ, 558, 133

McKee, C. F., \& Ostriker, J. P. 1977, ApJ, 218, 148
Mebold, U., Herbstmeier, U., Kalberla, P. M. W., et al. 1991, A\&A, 251, L1

Moos, H. W., Cash, W. C., Cowie, L. L., et al. 2000, ApJ, 538, L1

Morton, D. C., \& Noreau, L. 1994, ApJS, 95, 301

Neubig, M. M. S., \& Bruhweiler, F. C. 1997, AJ, 114, 1951

Osterbrock, D. E. 1959, PASP, 71, 23

Parravano, A., Hollenbach, D. J., \& McKee, C. F. 2003, ApJ, 584, 797

Péquignot, D., \& Aldrovandi, S. M. V. 1986, A\&A, 161, 169

Pfenniger, D., Combes, F., \& Martinet, L. 1994, A\&A, 285, 79

Pottasch, S. R. 1972, A\&A, 20, 245

Rachford, B. L., Snow, T. P., Tumlinson, J., et al. 2001, ApJ, 555, 839

Rachford, B. L., Snow, T. P., Tumlinson, J., et al. 2002, ApJ, 577, 221

Richter, P., de Boer, K. S., Widmann, H., et al. 1999, Nature, 402, 386

Richter, P., Sembach, K. R., \& Howk, J. C. 2003, A\&A, 405, 1013

Rollinde, E., Boissé, P., Federman, S. R., \& Pan, K. 2003, A\&A, 401, 215

Roueff, E., \& Zeippen, C. J. 2000, A\&AS, 142, 475

Russell, S. C., \& Dopita, M. A. 1992, ApJ, 384, 508

Sahnow, D. J., Moos, H. W., Ake, T. B., et al. 2000, ApJ, 538, L7

Savage, B. D., \& de Boer, K. S. 1981, ApJ, 243, 460

Savage, B. D., \& Sembach, K. R. 1991, ApJ, 379, 245

Savage, C., Apponi, A. J., Ziurys, L. M., \& Wyckoff, S. 2002, ApJ, 578, 211

Schectman, R. M., Federman, S. R., Beideck, D. J., \& Ellis, D. J. 1993 , ApJ, 406, 735

Schramm, D. N., \& Turner, M. S. 1998, Rev. Mod. Phys., 70, 303

Sembach, K. R., \& Savage, B. D. 1992, ApJS, 83, 147

Silvy, J. 1996, Ph.D. Université de Marseille

Snow, T. P., \& Cohen, J. G. 1974, ApJ, 194, 313

Snowden, S. L., \& Petre, R. 1994, ApJ, 436, L123

Songaila, A. 1981, ApJ, 248, 945

Sonneborn, G., André, M., Oliveira, C., et al. 2002, ApJS, 140, 51

Sonnentrucker, P., Friedman, S. D., Welty, D. E., York, D. G., \& Snow, T. P. 2002, ApJ, 576, 241

Sonnentrucker, P., Friedman, S. D., Welty, D. E., York, D. G., \& Snow, T. P. 2003, ApJ, 596, 350

Spitzer, L. J., \& Cochran, W. D. 1973, ApJ, 186, L23

Spitzer, L. J., \& Zweibel, E. G. 1974, ApJ, 191, L127

Tumlinson, J., Shull, J. M., Rachford, B. L., et al. 2002, ApJ, 566, 857

van der Tak, F. F. S., Schilke, P., Müller, H. S. P., et al. 2002, A\&A, 388, L53

Varshalovich, D. A., Ivanchik, A. V., Petitjean, P., Srianand, R., \& Ledoux, C. 2001, Astron. Lett., 27, 683

Vidal-Madjar, A., Andreani, P., Cristiani, S., et al. 1987, A\&A, 177, L17

Vladilo, G., Molaro, P., Monai, S., et al. 1993, A\&A, 274, 37

Wayte, S. R. 1990, ApJ, 355, 473

Welty, D. E., Morton, D. C., \& Hobbs, L. M. 1996, ApJS, 106, 533

Welty, D. E., Lauroesch, J. T., Blades, J. C., Hobbs, L. M., \& York, D. G. 1997, ApJ, 489, 672

Welty, D. E., Frisch, P. C., Sonneborn, G., \& York, D. G. 1999, ApJ, 512,636

Welty, D. E., \& Hobbs, L. M. 2001, ApJS, 133, 345

Wood, B. E., Linsky, J. L., Hébrard, G., et al. 2002, ApJS, 140, 91

Woodgate, B. E., Kimble, R. A., Bowers, C. W., et al. 1998, PASP, 110,1183

Woosley, S. E., \& Weaver, T. A. 1995, ApJS, 101, 181

Wright, M. C. H. 1970, MNRAS, 150, 271

Wright, E. L., \& Morton, D. C. 1979, ApJ, 227, 483 